\title{
Pacific
}

Journal of

Mathematics

\section{GEOMETRIC INEQUALITIES IN CARNOT GROUPS}

FRANCESCOPAOLO MONTEFALCONE

Volume 263 No. 1

May 2013 


\title{
GEOMETRIC INEQUALITIES IN CARNOT GROUPS
}

\author{
FRANCESCOPAOLO MONTEFALCONE
}

\section{Let $\mathbb{G}$ be a subriemannian $k$-step Carnot group of homogeneous dimension $Q$. We prove several geometric inequalities concerning smooth hypersur- faces (submanifolds of codimension one) immersed in $\mathbb{G}$, endowed with the $\mathrm{H}$-perimeter measure.}

1. Introduction

171

2. Isoperimetric constants and the first eigenvalue of $\mathscr{L}_{H S}$ on compact hypersurfaces

3. Two upper bounds on $\lambda_{1}$

4. Horizontal linear isoperimetric inequalities

5. A theorem about nonhorizontal graphs in 2-step Carnot groups 196

6. Local Poincaré-type inequality

\section{Introduction}

During the last years there has been an increasing interest in studying analysis and geometric measure theory in metric spaces (see [Ambrosio 2001; Ambrosio and Kirchheim 2000a; 2000b; Cheeger and Kleiner 2010; David and Semmes 1997; Garofalo and Nhieu 1996; Varopoulos et al. 1992] and bibliographic references therein, but this list is far from being exhaustive). In this regard, important examples of highly noneuclidean geometries are represented by the so-called Carnot-Carathéodory (or subriemannian) geometries; see [Capogna et al. 1994; Gromov 1996; Montgomery 2002; Pansu 1982; 1989; 2005; Strichartz 1986; Vershik and Gershkovich 1994]. In this context, Carnot groups play the role of modeling the tangent space (in a suitable generalized sense, which is related to the Gromov-Hausdorff convergence) of a subriemannian manifold; see [Gromov 1996; Montgomery 2002]. For this and many other reasons, Carnot groups are an intriguing field of research; see [Ambrosio et al. 2006; Balogh 2003; Balogh et al. 2009; Capogna et al. 2010; Cheng et al. 2005; Danielli et al. 2007; 2010; Franchi

Partially supported by the Fondazione CaRiPaRo Project "Nonlinear partial differential equations: models, analysis, and control-theoretic problems".

MSC2010: primary 49Q15; secondary 46E99, 43A80.

Keywords: Carnot groups, subriemannian geometry, hypersurfaces, geometric inequalities. 
et al. 2001; 2003a; 2003b; 2007; Hladky and Pauls 2008; Magnani 2002; Magnani and Vittone 2008; Montefalcone 2005; 2007a; Ritoré and Rosales 2008].

A $k$-step Carnot group $(\mathbb{G}, \bullet)$ is an $n$-dimensional, connected, simply connected, nilpotent, stratified Lie group (with respect to the group multiplication $\bullet$ ) whose Lie algebra $\mathfrak{g} \cong \mathbb{R}^{n}$ satisfies

$$
\mathfrak{g}=H_{1} \oplus \cdots \oplus H_{k}, \quad\left[H_{1}, H_{i-1}\right]=H_{i} \quad(i=2, \ldots, k), \quad H_{k+1}=\{0\} .
$$

We assume that $h_{i}=\operatorname{dim} H_{i}(i=1, \ldots, k)$ so that $n=\sum_{i=1}^{k} h_{i}$. Any Carnot group $\mathbb{G}$ has a 1-parameter family of dilations, adapted to the stratification, that makes it a homogeneous group, in the sense of Stein's definition [1993]. We refer the reader to Section 1.1 for a more detailed introduction to Carnot groups.

In this paper, we shall prove some geometric inequalities concerning smooth hypersurfaces immersed in a subriemannian $k$-step Carnot group $\mathbb{G}$ of homogeneous dimension $Q:=\sum_{i=1}^{k} i h_{i}$. We have to stress that hypersurfaces will be endowed with the so-called $H$-perimeter measure $\sigma_{H}^{n-1}$, which is a natural substitute for the intrinsic $(Q-1)$-dimensional CC Hausdorff measure. In Section 1.2, we will discuss some preliminary notions concerning homogeneous measures and the horizontal geometry of hypersurfaces. Then we will recall some tools which will be important in the sequel, such as a coarea-type formula and the horizontal integration by parts theory; see Section 1.3.

In Section 2 we will extend to this setting some isoperimetric-type constants, introduced in [Cheeger 1970] for compact riemannian manifolds and later studied in [Yau 1975].

In particular, we shall prove the validity of some global inequalities for smooth compact hypersurfaces with (or without) boundary, immersed into $\mathbb{G}$. Here, we would like to remark that there is a strong relationship between these inequalities and some eigenvalue problems related to the second-order differential operator $\mathscr{L}_{H S}$ (which is nothing but a horizontal version of the Laplace-Beltrami operator); see, more precisely, Definition 21 in Section 1.2.

Roughly speaking, after defining the isoperimetric constants (in purely geometric terms), we will show that they are equal to the infimum of some Rayleigh quotients. More precisely, let $S \subset \mathbb{G}$ be a smooth hypersurface and assume $\partial S \neq \varnothing$. Furthermore, set

$$
\operatorname{Isop}(S):=\inf \frac{\sigma_{H}^{n-2}(N)}{\sigma_{H}^{n-1}\left(S_{1}\right)},
$$

where $N \subset S$ is a smooth hypersurface of $S$ such that $N \cap \partial S=\varnothing$ and $S_{1}$ is the unique $(n-2)$-dimensional submanifold of $S$ such that $N=\partial S_{1}$. We have to stress that $\sigma_{H}^{n-1}$ and $\sigma_{H}^{n-2}$ denote homogeneous measures on $S_{1}$ and $N$, respectively. These measures can be thought of, respectively, as the $(Q-1)$-dimensional and 
the ( $Q-2)$-dimensional CC Hausdorff measures on $S_{1}$ and $N$; see Section 1.2. Then, it will be shown that

$$
\operatorname{Isop}(S)=\inf \frac{\int_{S}\left|\operatorname{grad}_{H S} \psi\right| \sigma_{H}^{n-1}}{\int_{S}|\psi| \sigma_{H}^{n-1}},
$$

where the infimum is taken over suitably smooth functions on $S$ such that $\left.\psi\right|_{\partial S}=0$. As mentioned, this constant is related to the first nonzero eigenvalue $\lambda_{1}$ of the following Dirichlet-type problem:

$$
\left\{\begin{aligned}
-\mathscr{L}_{H S} \psi & =\lambda \psi \\
\left.\psi\right|_{\partial S} & =0
\end{aligned}\right.
$$

see Definition 21. Indeed, we shall see that

$$
\lambda_{1} \geq \frac{(\operatorname{Isop}(S))^{2}}{4} ;
$$

see Corollary 28. Some similar results concerning another isoperimetric constant will be proved; see Theorem 30 and Corollary 31. The proofs of these results follow the scheme of the riemannian case, for which we refer the reader to [Yau 1975]; see also [Cheeger 1970] and [Chavel 1984; 1993]. We also remark that the main technical tool in the original proofs is the coarea formula.

In Section 3 we shall prove two geometric inequalities involving volume, $\mathrm{H}$ perimeter and the first eigenvalue of the operator $\mathscr{L}_{H S}$ on $S$. These results generalize an inequality of Chavel [1978] and an inequality of Reilly [1977], respectively.

In Section 4 we will extend to the Carnot setting some classical differentialgeometric results (such as linear isoperimetric inequalities); see, for instance, [Burago and Zalgaller 1988] and references therein. The starting point is an integral formula very similar to the euclidean Minkowski formula; see Corollary 20 for a precise statement. In particular, we will show that

$$
(h-1) \sigma_{H}^{n-1}(S) \leq R\left(\int_{S}\left(\left|\mathscr{H}_{H}\right|+\left|C_{H} v_{H}\right|\right) \sigma_{H}^{n-1}+\sigma_{H}^{n-2}(\partial S)\right),
$$

where $S \subset \mathbb{G}$ is a compact hypersurface with boundary and $R$ denotes the radius of a homogeneous $\varrho$-ball circumscribed about $S$. From this linear (isoperimetric) inequality, it is possible to infer some geometric consequences and, among them, we prove a weak monotonicity inequality for the $H$-perimeter; see Section 4.1, Proposition 38.

Section 5 contains a theorem about nonhorizontal graphs in 2-step Carnot groups. This generalizes a classical result of Heinz [1955]; see also [Chern 1965].

Let us describe this result in the simpler case of the Heisenberg group $\mathbb{M}^{1}$. So let $S \subset \mathbb{M}^{1}$ be a $T$-graph associated with a function $t=f(x, y)$ of class $C^{2}$ over the 
$x y$-plane. If the horizontal mean curvature $\mathscr{H}_{H}$ of $S$ satisfies a bound $\left|\mathscr{H}_{H}\right| \geq C>0$, then

$$
C \mathscr{H}_{\mathrm{Eu}}^{2}\left(\mathscr{P}_{x y}(\mathcal{U})\right) \leq \mathscr{H}_{\mathrm{Eu}}^{1}\left(\mathscr{P}_{x y}(\partial \mathcal{U})\right)
$$

for every $C^{1}$-smooth relatively compact open set $U \subset S$, where $\mathscr{H}_{\mathrm{Eu}}^{i}(i=1,2)$ is the usual $i$-dimensional euclidean Hausdorff measure and $\mathscr{P}_{x y}$ is the orthogonal projection onto the $x y$-plane. Hence, taking $U:=S \cap C_{r}(\mathscr{T})$, where $C_{r}(\mathscr{T})$ denotes a vertical cylinder of radius $r$ around the $T$-axis of $\mathbb{U}^{1}$, yields

$$
r \leq \frac{2}{C}
$$

for every $r>0$. It follows that any entire $x y$-graph of class $C^{2}$, having either constant or only bounded horizontal mean curvature $\mathscr{H}_{H}$, must be necessarily a $H$-minimal surface. An analogous result holds true in the framework of step 2 Carnot groups; see Theorem 42.

In Section 6 we shall study some (local) Poincaré-type inequalities, depending on the local geometry of the hypersurface $S$ and, more precisely, on its characteristic set $C_{S}$; see Theorems 44 and 45 .

For instance, let $S \subset \mathbb{G}$ be a $C^{2}$-smooth hypersurface with bounded horizontal mean curvature $\mathscr{H}_{H}$. Then, we shall prove that for every $x \in S$ there exists $R_{0} \leq$ $\operatorname{dist}_{\varrho}(x, \partial S)$ (which explicitly depends on $C_{S}$ ) such that

$$
\left(\int_{S_{R}}|\psi|^{p} \sigma_{H}^{n-1}\right)^{\frac{1}{p}} \leq C_{p} R\left(\int_{S_{R}}\left|\operatorname{grad}_{H S} \psi\right|^{p} \sigma_{H}^{n-1}\right)^{\frac{1}{p}}, \quad p \in[1,+\infty[,
$$

for all $\psi \in C_{0}^{1}\left(S_{R}\right)$ and all $R \leq R_{0}$, where $S_{R}:=S \cap B_{\varrho}(x, R)$.

These results are obtained by means of elementary "linear" estimates starting from the horizontal integration by parts formula, together with a simple analysis of the role played by the characteristic set. Finally, in Section 6.1 we will prove the validity of a Caccioppoli-type inequality for weak solutions of the operator $\mathscr{L}_{H S}$.

1.1. Carnot groups. A $k$-step Carnot group $(\mathbb{G}, \bullet)$ is a finite-dimensional connected, simply connected, nilpotent and stratified Lie group with respect to a polynomial group law $\bullet$. The Lie algebra $\mathfrak{g} \cong \mathbb{R}^{n}$ fulfills the conditions $\mathfrak{g}=H_{1} \oplus \cdots \oplus H_{k}$, $\left[H_{1}, H_{i-1}\right]=H_{i}$ for all $i=2, \ldots, k+1, H_{k+1}=\{0\}$, where $[\cdot, \cdot]$ denotes the Lie bracket and each $H_{i}$ is a vector subspace of $\mathfrak{g}$. In particular, we denote by 0 the identity of $\mathbb{G}$ and assume that $\mathfrak{g} \cong T_{0} \mathbb{G}$. We also use the notation $H:=H_{1}$ and $V:=H_{2} \oplus \cdots \oplus H_{k}$. The subspaces $H$ and $V$ are smooth subbundles of $T \mathbb{G}$ called horizontal and vertical, respectively.

Notation 1. Throughout this paper, we denote by $\mathscr{P}_{H_{i}}: T G \rightarrow H_{i}$ the orthogonal projection map from $T \mathbb{G}$ onto $H_{i}$ for any $i=1, \ldots, k$. In particular, we set 
$\mathscr{P}_{H}:=\mathscr{P}_{H_{i}}$. Analogously, we set $\mathscr{P}_{V}: T \mathbb{G} \rightarrow V$ to denote the orthogonal projection map from $T \mathbb{G}$ onto $V$.

Let $h_{i}:=\operatorname{dim} H_{i}$ for any $i=1, \ldots, k$. Set $n_{0}:=0$ and $n_{i}:=\sum_{j=1}^{i} h_{j}$ for any $i=1, \ldots, k$. Note that $n_{1}=h_{1}, n_{2}=h_{1}+h_{2}, \ldots$, and $n_{k}=n$.

Notation 2. Throughout this paper, we set $I_{H_{i}}:=\left\{n_{i-1}+1, \ldots, n_{i}\right\}$ for any $i=1, \ldots, k$. We also set $I_{V}:=\left\{h_{1}+1, \ldots, n\right\}$ and use Greek letters $\alpha, \beta, \gamma, \ldots$, for indices in $I_{V}$. For the sake of simplicity, we set $h:=h_{1}$ and $I_{H}:=I_{H_{1}}$.

The horizontal bundle $H$ is generated by a frame $X_{H}:=\left\{X_{1}, \ldots, X_{h}\right\}$ of leftinvariant vector fields. This frame can be completed to a global graded, left-invariant frame $\underline{X}:=\left\{X_{1}, \ldots, X_{n}\right\}$ for $T \mathbb{G}$. Note that the standard basis $\left\{\mathrm{e}_{i}: i=1, \ldots, n\right\}$ of $\mathbb{R}^{n}$ can be relabeled to be graded or adapted to the stratification. Any leftinvariant vector field of the frame $\underline{X}$ is given by $X_{i}(x)=L_{x *} \mathrm{e}_{i}(i=1, \ldots, n)$, where $L_{x *}$ denotes the differential of the left-translation $L_{x}$, defined by $L_{x} y:=x \bullet y$ for all $y \in \mathbb{G}$. We also fix a euclidean metric on $\mathfrak{g}=T_{0} \mathbb{G}$ such that $\left\{\mathrm{e}_{i}: i=1, \ldots, n\right\}$ is an orthonormal basis. This metric $g=\langle\cdot, \cdot\rangle$ extends to the whole tangent bundle by left-translations and makes $\underline{X}$ an orthonormal left-invariant frame. Therefore $(\mathbb{G}, g)$ is a riemannian manifold.

Let $\exp : \mathfrak{g} \rightarrow \mathbb{G}$ be the exponential map. Hereafter, we will use exponential coordinates of the first kind; see [Varadarajan 1974, Chapter 2, p. 88].

As for the case of nilpotent Lie groups, the multiplication $\bullet$ of $\mathbb{G}$ is uniquely determined by the "structure" of the Lie algebra $\mathfrak{g}$. This is the content of the BakerCampbell-Hausdorff formula; see [Corwin and Greenleaf 1990]. More precisely,

$$
\exp (X) \cdot \exp (Y)=\exp (X \star Y) \text { for all } X, Y \in \mathfrak{g},
$$

where $\star: \mathfrak{g} \times \mathfrak{g} \rightarrow \mathfrak{g}$ denotes the Baker-Campbell-Hausdorff product, given by

$$
\begin{aligned}
X \star Y=X+Y+\frac{1}{2}[X, Y]+\frac{1}{12}[X,[X, Y]]-\frac{1}{12}[Y,[X, Y]] \\
+ \text { brackets of length } \geq 3 .
\end{aligned}
$$

Using exponential coordinates and (1), the group multiplication • turns out to be polynomial and explicitly computable; see [Corwin and Greenleaf 1990]. Moreover, $0=\exp (0, \ldots, 0)$ and the inverse of $x \in \mathbb{G}\left(x=\exp \left(x_{1}, \ldots, x_{n}\right)\right)$ is $x^{-1}=\exp \left(-x_{1}, \ldots,-x_{n}\right)$.

A subriemannian metric $g_{H}$ is a symmetric positive bilinear form on the horizontal bundle $H$. The $C C$-distance $d_{\mathrm{CC}}(x, y)$ between $x, y \in \mathbb{G}$ is given by

$$
d_{\mathrm{CC}}(x, y):=\inf \int \sqrt{g_{H}(\dot{\gamma}, \dot{\gamma})} d t
$$

where the infimum is taken over all piecewise-smooth horizontal paths $\gamma$ joining $x$ to $y$. Later, we shall choose $g_{H}:=g_{\mid H}$. 
Carnot groups are homogeneous groups; that is, they admit a 1-parameter group of automorphisms $\delta_{t}: \mathbb{G} \rightarrow \mathbb{G}(t \geq 0)$ defined by $\delta_{t} x:=\exp \left(\sum_{j, i_{j}} t^{j} x_{i_{j}} \mathrm{e}_{i_{j}}\right)$, where $x=\exp \left(\sum_{j, i_{j}} x_{i_{j}} \mathrm{e}_{i_{j}}\right) \in \mathbb{G}$. As already said, the homogeneous dimension of $\mathbb{G}$ is the integer $Q:=\sum_{i=1}^{k} i h_{i}$ coinciding with the Hausdorff dimension of $\left(\mathbb{G}, d_{\mathrm{CC}}\right)$ as a metric space; see [Montgomery 2002].

We recall that a continuous distance $\varrho: \mathbb{G} \times \mathbb{G} \rightarrow \mathbb{R}_{+} \cup\{0\}$ is a homogeneous distance if, and only if, $\varrho(x, y)=\varrho(z \bullet x, z \bullet y)$ for all $x, y, z \in \mathbb{G} ; \quad \varrho\left(\delta_{t} x, \delta_{t} y\right)=t \varrho(x, y)$ for all $t \geq 0$.

The structural constants of $\mathfrak{g}$ (see [Chavel 1993]) associated with the frame $\underline{X}$ are defined by $C_{i j}^{r}:=\left\langle\left[X_{i}, X_{j}\right], X_{r}\right\rangle$ for all $i, j, r=1, \ldots, n$. They are skewsymmetric and satisfy Jacobi's identity. The stratification of the Lie algebra $\mathfrak{g}$ implies a fundamental "structural" property of Carnot groups: if $X_{i} \in H_{l}, X_{j} \in H_{m}$, then $\left[X_{i}, X_{j}\right] \in H_{l+m}$. Note that, if $i \in I_{H_{s}}$ and $j \in I_{H_{r}}$, then

$$
C_{i j}^{m} \neq 0 \Longrightarrow m \in I_{H_{s+r}} \text {. }
$$

Equivalently, if $C_{i j}^{r} \neq 0$, then $\operatorname{ord}(i)+\operatorname{ord}(j)=\operatorname{ord}(r)$, where ord $:\{1, \ldots, n\} \rightarrow$ $\{1, \ldots, k\}$ is the function defined as $\operatorname{ord}(l)=i \Longleftrightarrow l \in I_{H_{i}}$.

Notation 3. Henceforth, we shall set

- $C_{H}^{\alpha}:=\left[C_{i j}^{\alpha}\right]_{i, j=1, \ldots, h} \in M_{h \times h}(\mathbb{R})$ for all $\alpha \in I_{H_{2}}=\left\{h+1, \ldots, h+h_{2}\right\}$;

- $C^{\alpha}:=\left[C_{i j}^{\alpha}\right]_{i, j=1, \ldots, n} \in M_{n \times n}(\mathbb{R})$ for all $\alpha \in I_{V}=\{h+1, \ldots, n\}$.

Remark 4. It is important to observe that (2) immediately implies that the matrices just defined are the only ones which can be nonzero.

Let us define the left-invariant coframe $\underline{\omega}:=\left\{\omega_{i}: i=1, \ldots, n\right\}$ dual to $\underline{X}$; i.e., $\omega_{i}=X_{i}^{*}$ for every $i=1, \ldots, n$. The left-invariant 1 -forms $\omega_{i}$ for $i=1, \ldots, n$ are uniquely determined by the condition $\omega_{i}\left(X_{j}\right)=\left\langle X_{i}, X_{j}\right\rangle=\delta_{i}^{j}$ for all $i, j=1, \ldots, n$, where $\delta_{i}^{j}$ denotes Kronecker delta.

Definition 5. We shall denote by $\nabla$ the unique left-invariant Levi-Civita connection on $\mathbb{G}$ associated with the left-invariant metric $g=\langle\cdot, \cdot\rangle$. Moreover, if $X, Y \in$ $\mathfrak{X}(H):=C^{\infty}(\mathbb{G}, H)$, we shall set

$$
\nabla_{X}^{H} Y:=\mathscr{P}_{H}\left(\nabla_{X} Y\right)
$$

Let $\underline{X}=\left\{X_{1}, \ldots, X_{n}\right\}$ be the global left-invariant frame on $T \mathbb{G}$. Then

$$
\nabla_{X_{i}} X_{j}=\frac{1}{2} \sum_{r=1}^{n}\left(C_{i j}^{r}-C_{j r}^{i}+C_{r i}^{j}\right) X_{r} \quad \text { for all } i, j=1, \ldots, n
$$

see, for instance, [Milnor 1976, Section 5, pp. 310-311]. Furthermore, we stress 
that $\nabla^{H}$ is a partial connection, called horizontal H-connection; see [Ge 1992] or [Koiller et al. 2001]; see also [Montefalcone 2007a] and references therein. Using Definition 5 together with (3) and (2), it is not difficult to show the following:

- $\nabla^{H}$ is flat; i.e.,

$$
\nabla_{X_{i}}^{H} X_{j}=0 \text { for all } i, j \in I_{H}
$$

- $\nabla^{H}$ is compatible with the subriemannian metric $g_{H}$; i.e.,

$$
X\langle Y, Z\rangle=\left\langle\nabla_{X}^{H} Y, Z\right\rangle+\left\langle Y, \nabla_{X}^{H} Z\right\rangle \text { for all } X, Y, Z \in \mathfrak{X}(H) ;
$$

- $\nabla^{H}$ is torsion-free; i.e.,

$$
\nabla_{X}^{H} Y-\nabla_{Y}^{H} X-\mathscr{P}_{H}[X, Y]=0 \quad \text { for all } X, Y \in \mathfrak{X}(H) .
$$

Definition 6. If $\psi \in C^{\infty}(\mathbb{G})$ we define the horizontal gradient of $\psi$ as the unique horizontal vector field $\operatorname{grad}_{H} \psi$ such that $\left\langle\operatorname{grad}_{H} \psi, X\right\rangle=d \psi(X)=X \psi$ for every $X \in \mathfrak{X}(H)$. The horizontal divergence of $X \in \mathfrak{X}(H), \operatorname{div}_{H} X$, is defined, at each point $x \in \mathbb{G}$, by

$$
\operatorname{div}_{H} X(x):=\operatorname{Trace}\left(Y \rightarrow \nabla_{Y}^{H} X\right)(x) \quad\left(Y \in H_{x}\right) .
$$

For any $Y=\sum_{j \in I_{H}} y_{j} X_{j} \in \mathfrak{X}(H)$, we denote by $\mathscr{J}_{H} Y$ the horizontal Jacobian matrix of $Y$; i.e.,

$$
\mathscr{J}_{H} Y:=\left[X_{i}\left(y_{j}\right)\right]_{j, i \in I_{H}} .
$$

Example 7 (Heisenberg group $\mathbb{H}^{n}(n \geq 1)$ ). The Lie algebra $\mathfrak{h}_{n} \cong \mathbb{R}^{2 n+1}$ of the $n$-th Heisenberg group $\mathbb{\boxplus}^{n}$ can be described by means of a left-invariant frame $\underline{Z}:=$ $\left\{X_{1}, Y_{1}, \ldots, X_{i}, Y_{i}, \ldots, X_{n}, Y_{n}, T\right\}$, where, at each $p=\exp \left(x_{1}, y_{1}, x_{2}, y_{2}, \ldots\right.$, $\left.x_{n}, y_{n}, t\right) \in \mathbb{H}^{n}$, we have set $X_{i}(p):=\partial / \partial x_{i}-\frac{1}{2} y_{i} \partial / \partial t, Y_{i}(p):=\partial / \partial y_{i}+\frac{1}{2} x_{i} \partial / \partial t$ for every $i=1, \ldots, n ; T(p):=\partial / \partial t$. One has $\left[X_{i}, Y_{i}\right]=T$ for every $i=1, \ldots, n$, and all other commutators vanish, so that $T$ is the center of $\mathfrak{h}_{n}$ and $\mathfrak{h}_{n}$ turns out to be a nilpotent and stratified Lie algebra of step 2; i.e., $\mathfrak{h}_{n}=H \oplus H_{2}$. The structural constants of $\mathfrak{h}_{n}$ are described by the skew-symmetric $(2 n \times 2 n)$-matrix

$$
C_{H}^{2 n+1}:=\left|\begin{array}{rrrrr}
0 & 1 & . & 0 & 0 \\
-1 & 0 & . & 0 & 0 \\
. & . & . & . & . \\
0 & 0 & . & 0 & 1 \\
0 & 0 & . & -1 & 0
\end{array}\right| .
$$

1.2. Hypersurfaces. The (riemannian) left-invariant volume form of any Carnot group $\mathbb{G}$ is defined as $\sigma_{R}^{n}:=\bigwedge_{i=1}^{n} \omega_{i} \in \bigwedge^{n}\left(T^{*} \mathbb{G}\right)$. By integration of the $n$-form $\sigma_{R}^{n}$, one obtains the Haar measure of $\mathbb{G}$, which equals the push-forward of the $n$ dimensional Lebesgue measure $\mathscr{L}^{n}$ on $\mathfrak{g} \cong \mathbb{R}^{n}$. The symbols $\mathscr{H}_{\mathrm{CC}}^{s}, \mathscr{H}_{\mathrm{Eu}}^{s}$ will denote 
the intrinsic CC $s$-dimensional Hausdorff measure and the euclidean $s$-dimensional Hausdorff measure, respectively. (Sometimes we will use the notation $\sigma_{R}^{n}=V_{\mathrm{ol}^{n}}{ }^{\text {). }}$. Let $S \subset \mathbb{G}$ be a hypersurface (i.e., a codimension 1 submanifold of $\mathbb{G}$ ) of class $C^{i}$ $(i \geq 1)$. Let $v$ denote the (riemannian) unit normal vector along $S$. Then $x \in S$ is a characteristic point if and only if $\operatorname{dim} H_{x}=\operatorname{dim}\left(H_{x} \cap T_{x} S\right)$. The characteristic set of $S$ is given by $C_{S}:=\left\{x \in S: \operatorname{dim} H_{x}=\operatorname{dim}\left(H_{x} \cap T_{x} S\right)\right\}$. In other words, a point $x \in S$ is noncharacteristic (hereafter abbreviated as NC) if and only if $H$ is transversal to $S$ at $x$. Hence, one has $C_{S}:=\left\{x \in S:\left|\mathscr{P}_{H} v(x)\right|=0\right\}$, where $\mathscr{P}_{H}$ denotes orthogonal projection onto $H$. It is of fundamental importance that the $(Q-1)$-dimensional CC Hausdorff measure of the characteristic set $C_{S}$ vanishes; i.e., $\mathscr{H}_{\mathrm{CC}}^{Q-1}\left(C_{S}\right)=0$; see, for instance, Theorem 6.6.2 in [Magnani 2002]. We also stress that if $S$ is a hypersurface of class $C^{2}$, then precise estimates of the riemannian Hausdorff dimension of $C_{S}$ can be found in [Balogh et al. 2010]; see also [Balogh 2003] for the case of the Heisenberg group $\mathbb{H}^{n}(n \geq 1)$.

The $(n-1)$-dimensional riemannian measure along $S$ is defined by integration of the $(n-1)$-differential form $\left.\sigma_{R}^{n-1}\left\llcorner S:=(v\lrcorner \sigma_{R}^{n}\right)\right|_{S}$, where $\lrcorner$ denotes the "contraction" operator on differential forms; see [Federer 1969]. We recall that \lrcorner$: \bigwedge^{k}\left(T^{*} \mathbb{G}\right) \rightarrow \bigwedge^{k-1}\left(T^{*} \mathbb{G}\right)$ is defined, for $X \in T \mathbb{G}$ and $\alpha \in \bigwedge^{k}\left(T^{*} \mathbb{G}\right)$, by setting $(X\lrcorner \alpha)\left(Y_{1}, \ldots, Y_{k-1}\right):=\alpha\left(X, Y_{1}, \ldots, Y_{k-1}\right)$.

At each NC point $x \in S \backslash C_{S}$ the unit H-normal is defined as

$$
v_{H}:=\frac{\mathscr{P}_{H} \nu}{\left|\mathscr{P}_{H} \nu\right|} .
$$

Similarly to the riemannian case, we define an $(n-1)$-differential form $\sigma_{H}^{n-1} \in$ $\bigwedge^{n-1}\left(T^{*} S\right)$ by setting

$$
\left.\sigma_{H}^{n-1}\left\llcorner S:=\left(v_{H}\right\lrcorner \sigma_{R}^{n}\right)\right|_{S} .
$$

By integration of $\sigma_{H}^{n-1}\llcorner S$, one gets a left-invariant and $(Q-1)$-homogeneous measure, which is called $H$-perimeter measure. This measure can be extended to the whole of $S$ by setting $\sigma_{H}^{n-1}\left\llcorner C_{S}=0\right.$. Note that $\sigma_{H}^{n-1}\left\llcorner S=\left|\mathscr{P}_{H} \nu\right| \sigma_{R}^{n-1}\llcorner S\right.$. Furthermore, denoting by $\mathscr{S}_{\mathrm{CC}}^{Q-1}$ the $(Q-1)$-dimensional spherical intrinsic CC Hausdorff measure (i.e., associated with the CC-distance $d_{\mathrm{CC}}$ ), then

$$
\sigma_{H}^{n-1}(S \cap B)=k\left(v_{H}\right) \mathscr{P}_{\mathrm{CC}}^{Q-1}\llcorner(S \cap B) \quad \text { for all } B \in \mathscr{B} \text { or }(\mathbb{G}),
$$

where the density-function $k\left(v_{H}\right)$, called metric factor, explicitly depends on $v_{H}$ and $d_{\mathrm{CC}}$; see [Magnani 2002].

At each NC point $x \in S \backslash C_{S}$, the horizontal tangent bundle $H S:=H \cap T S \subset T S$ and the horizontal normal bundle $v_{H} S \subset H$ split the horizontal bundle $H$ into an orthogonal direct sum; i.e., $H=v_{H} \oplus H S$. The stratification of $\mathfrak{g}$ induces a 
stratification of $T S:=\oplus_{i=1}^{k} H_{i} S$, where we have set $H S:=H_{1} S$; see [Gromov 1996]. Note that at any characteristic point $x \in C_{S}$ one has $H_{x}=H_{x} S$, so that

$$
\operatorname{dim}\left(H_{x} S\right)=\left\{\begin{array}{cl}
h-1 & \text { if } x \in S \backslash C_{S}, \\
h & \text { if } x \in C_{S} .
\end{array}\right.
$$

Notation 8. Throughout this paper, we denote by $\mathscr{P}_{H S}: T S \rightarrow H S$ the orthogonal projection map from $T S$ onto $H S$.

Now let $S \subset \mathbb{G}$ be a hypersurface of class $C^{2}$ and let $\nabla^{T S}$ denote the induced connection on $S$ from $\nabla$. The tangential connection $\nabla^{T S}$ induces a partial connection on $H S$ defined by

$$
\nabla_{X}^{H S} Y:=\mathscr{P}_{H S}\left(\nabla_{X}^{T S} Y\right) \text { for all } X, Y \in \mathfrak{X}^{1}(H S):=C^{1}(S, H S) .
$$

It turns out that

$$
\nabla_{X}^{H S} Y=\nabla_{X}^{H} Y-\left\langle\nabla_{X}^{H} Y, v_{H}\right\rangle v_{H} \quad \text { for every } X, Y \in \mathfrak{X}^{1}(H S) ;
$$

see [Montefalcone 2007a].

Definition 9 (see [Montefalcone 2007a]). We call $H S$-gradient of $\psi \in C^{1}(S)$ the unique horizontal tangent vector field $\operatorname{grad}_{H S} \psi$ such that

$$
\left\langle\operatorname{grad}_{H S} \psi, X\right\rangle=d \psi(X)=X \psi \quad \text { for all } X \in \mathfrak{X}^{1}(H S) .
$$

We denote by $\operatorname{div}_{H S}$ the $H S$-divergence; i.e., if $X \in \mathfrak{X}^{1}(H S)$ and $x \in S$, then

$$
\operatorname{div}_{H S} X(x):=\operatorname{Trace}\left(Y \rightarrow \nabla_{Y}^{H S} X\right)(x) \quad\left(Y \in H_{x} S\right) .
$$

The $H S$-Laplacian $\Delta_{H S}$ is the second-order differential operator defined as

$$
\Delta_{H S} \psi:=\operatorname{div}_{H S}\left(\operatorname{grad}_{H S} \psi\right) \text { for every } \psi \in C^{2}(S) .
$$

The horizontal second fundamental form of $S \backslash C_{S}$ is the map given by

$$
B_{H}(X, Y):=\left\langle\nabla_{X}^{H} Y, v_{H}\right\rangle \text { for all } X, Y \in \mathfrak{X}^{1}(H S) .
$$

The horizontal mean curvature $\mathscr{H}_{H}$ is the trace of $B_{H}$; i.e., $\mathscr{H}_{H}:=\operatorname{Tr} B_{H}=-\operatorname{div}_{H} v_{H}$.

It is worth observing that the $H S$-connection admits, in general, a nonzero torsion because $B_{H}$ is not symmetric; see [Montefalcone 2007a].

Definition 10. Let $U \subseteq S$ be an open set. We shall denote by $C_{H S}^{i}(U)(i=1,2)$ the space of functions whose $H S$-derivatives up to $i$-th order are continuous on $U$.

We stress that the previous definitions concerning the horizontal second fundamental form $B_{H}(\cdot, \cdot)$ and the $H S$-connection can also be reformulated by using the function space $C_{H S}^{i}(U)(i=1,2)$ and, more precisely, by replacing $\mathfrak{X}^{1}(H S)=C^{1}(S, H S)$ with $\mathfrak{X}_{H S}^{1}(H S):=C_{H S}^{1}(S, H S)$. 
Let $S \subset \mathbb{G}$ be a hypersurface of class $C^{i}(i \geq 1)$ and let $v$ be the outward-pointing unit normal vector field along $S$. We need to define some important geometric objects. To this end, we first note that $v=\mathscr{P}_{H} v+\mathscr{P}_{V} v$. By using the left-invariant frame $\underline{X}=\left\{X_{1}, \ldots, X_{n}\right\}$, we see that $\mathscr{P}_{V} v=\sum_{\alpha \in I_{V}} v_{\alpha} X_{\alpha}$, where $v_{\alpha}:=\left\langle v, X_{\alpha}\right\rangle$; see Notation 2.

Notation 11. Hereafter we shall set

- $\varpi_{\alpha}:=\frac{v_{\alpha}}{\left|\mathscr{P}_{H} \nu\right|}$ for all $\alpha \in I_{V}$;

- $\varpi:=\sum_{\alpha \in I_{V}} \varpi_{\alpha} X_{\alpha}$;

- $C_{H}:=\sum_{\alpha \in I_{H_{2}}} \varpi_{\alpha} C_{H}^{\alpha}$;

see, for instance, Notation 3 and Remark 4.

1.3. Other tools. Let $S \subset \mathbb{G}$ be a hypersurface of class $C^{i}(i \geq 1)$. Let $\partial S$ be an $(n-2)$-dimensional submanifold of $S$ of class $C^{1}$, oriented by the outward pointing unit normal vector $\eta \in T S \cap \operatorname{Nor}(\partial S)$. We shall denote by $\sigma_{R}^{n-2}$ the riemannian measure on $\partial S$; i.e., $\left.\sigma_{R}^{n-2}\left\llcorner\partial S=(\eta\lrcorner \sigma_{R}^{n-1}\right)\right|_{\partial S}$. In particular, note that $\left.(X\lrcorner \sigma_{H}^{n-1}\right)\left.\right|_{\partial S}=\langle X, \eta\rangle\left|\mathscr{P}_{H} \nu\right| \sigma_{R}^{n-2}\left\llcorner\partial S\right.$ for every $X \in \mathfrak{X}^{1}(T S):=C^{1}(S, T S)$. The unit $H S$-normal along $\partial S$ is given by $\eta_{H S}:=\mathscr{P}_{H S} \eta /\left|\mathscr{P}_{H S} \eta\right|$. In this way, we can define a homogeneous $(n-2)$-dimensional measure $\sigma_{H}^{n-2} \in \bigwedge^{n-2}\left(T^{*} \partial S\right)$ by setting $\left.\sigma_{H}^{n-2}\left\llcorner\partial S:=\left(\eta_{H S}\right\lrcorner \sigma_{H}^{n-1}\right)\right|_{\partial S}$. It follows that

$$
\sigma_{H}^{n-2}\left\llcorner\partial S=\left|\mathscr{P}_{H} \nu\right|\left|\mathscr{P}_{H S} \eta\right| \sigma_{R}^{n-2}\llcorner\partial S\right.
$$

and that $\left.(X\lrcorner \sigma_{H}^{n-1}\right)\left.\right|_{\partial S}=\left\langle X, \eta_{H S}\right\rangle \sigma_{H}^{n-2}\left\llcorner\partial S\right.$ for all $X \in \mathfrak{X}^{1}(H S):=C^{1}(S, H S)$.

Now let $v \wedge \eta \in \Lambda^{2}(T S)$ be a unit 2-vector orienting $\partial S$, where $v \in \operatorname{Nor}(S)$ and $\eta \in T S \cap \operatorname{Nor}(\partial S)$. Then, the characteristic set of $\partial S$ is defined as

$$
C_{\partial S}:=\left\{p \in \partial S:\left|\mathscr{P}_{H}(\nu \wedge \eta)\right|=0\right\},
$$

where the orthogonal projection operator $\mathscr{P}_{H}$ is extended to 2-vectors in the standard way.

Proposition 12. Let $S \subset \mathbb{G}$ be a compact hypersurface of class $C^{1}$ and let $\phi \in$ $C_{H S}^{1}(S)$. Then

$$
\int_{S}\left|\operatorname{grad}_{H S} \phi(x)\right| \sigma_{H}^{n-1}(x)=\int_{\mathbb{R}} \sigma_{H}^{n-2}\left\{\phi^{-1}[s] \cap S\right\} d s .
$$

Proof. This formula follows from the riemannian coarea formula; see [Burago and Zalgaller 1988], [Chavel 2001] or [Montefalcone 2009]. We have

$$
\int_{S} \phi(x)\left|\operatorname{grad}_{T S} \varphi(x)\right| \sigma_{R}^{n-1}(x)=\int_{\mathbb{R}} d s \int_{\varphi^{-1}[s] \cap S} \phi(y) \sigma_{R}^{n-2}(y)
$$


for every $\phi \in L^{1}\left(S, \sigma_{R}^{n-1}\right)$; see [Burago and Zalgaller 1988; Chavel 2001]. Choosing

$$
\phi=\frac{\left|\operatorname{grad}_{H S} \varphi\right|}{\left|\operatorname{grad}_{T S} \varphi\right|}\left|\mathscr{P}_{H} \nu\right|
$$

yields

$$
\int_{S} \phi\left|\operatorname{grad}_{T S} \varphi\right| \sigma_{R}^{n-1}=\int_{S} \frac{\left|\operatorname{grad}_{H S} \varphi\right|}{\left|\operatorname{grad}_{T S} \varphi\right|}\left|\operatorname{grad}_{T S} \varphi\right| \underbrace{\left|\mathscr{P}_{H} \nu\right| \sigma_{R}^{n-1}}_{=\sigma_{H}^{n-1}}=\int_{S}\left|\operatorname{grad}_{H S} \varphi\right| \sigma_{H}^{n-1} .
$$

The (riemannian) unit normal $\eta$ along $\varphi^{-1}[s]$ is given by $\eta=\operatorname{grad}_{T S} \varphi /\left|\operatorname{grad}_{T S} \varphi\right|$. Hence $\left|\mathscr{P}_{H S} \eta\right|=\left|\operatorname{grad}_{H S} \varphi\right| /\left|\operatorname{grad}_{T S} \varphi\right|$ and it turns out that

$$
\begin{aligned}
\int_{\mathbb{R}} d s \int_{\varphi^{-1}[s] \cap S} \phi(y) \sigma_{R}^{n-2} & =\int_{\mathbb{R}} d s \int_{\varphi^{-1}[s] \cap S} \frac{\left|\operatorname{grad}_{H S} \varphi\right|}{\left|\operatorname{grad}_{T S} \varphi\right|}\left|\mathscr{P}_{H} \nu\right| \sigma_{R}^{n-2} \\
& =\int_{\mathbb{R}} d s \int_{\varphi^{-1}[s] \cap S} \underbrace{\left|\mathscr{P}_{H S} \eta\right|\left|\mathscr{P}_{H} \nu\right| \sigma_{R}^{n-2}}_{=\sigma_{H}^{n-2}} \\
& =\int_{\mathbb{R}} d s \int_{\varphi^{-1}[s] \cap S} \sigma_{H}^{n-2} .
\end{aligned}
$$

Below, we recall a basic integration by parts formula for horizontal vector fields; see [Montefalcone 2007a].

Definition 13. Let $\mathscr{D}_{H S}: \mathfrak{X}_{H S}^{1}(H S) \rightarrow C(S)$ be the first-order differential operator given by

$$
\mathscr{D}_{H S} X:=\operatorname{div}_{H S} X+\left\langle C_{H} v_{H}, X\right\rangle \text { for all } X \in \mathfrak{X}_{H S}^{1}(H S)\left(:=C_{H S}^{1}(S, H S)\right) \text {. }
$$

Furthermore, let $\mathscr{L}_{H S}: C_{H S}^{2}(S) \rightarrow C(S)$ be the second-order differential operator given by

$$
\mathscr{L}_{H S} \varphi:=\Delta_{H S} \varphi+\left\langle C_{H} v_{H}, \operatorname{grad}_{H S} \varphi\right\rangle \quad \text { for all } \varphi \in C_{H S}^{2}(S) ;
$$

see Definition 9 and Notation 11.

The horizontal matrix $C_{H}$ is a key object, related to the skew-symmetric part of the horizontal second fundamental form $B_{H}$. Note that $\mathscr{D}_{H S}(\varphi X)=\varphi \mathscr{D}_{H S} X+$ $\left\langle\operatorname{grad}_{H S} \varphi, X\right\rangle$ for every $X \in \mathfrak{X}_{H S}^{1}(H S)$ and every $\varphi \in C_{H S}^{1}(S)$. Moreover, one has $\mathscr{L}_{H S} \varphi=\mathscr{D}_{H S}\left(\operatorname{grad}_{H S} \varphi\right)$ for every $\varphi \in C_{H S}^{2}(S)$. These definitions are motivated by Theorem 3.17, Corollary 3.18 and Corollary 3.19 in [Montefalcone 2007a].

Theorem 14 (see [Montefalcone 2007a]). Let $S$ be a compact NC hypersurface of class $C^{2}$ with boundary $\partial S$ of class $C^{1}$. Then

$$
\int_{S} \mathscr{D}_{H S} X \sigma_{H}^{n-1}=-\int_{S} \mathscr{H}_{H}\left\langle X, v_{H}\right\rangle \sigma_{H}^{n-1}+\int_{\partial S}\left\langle X, \eta_{H S}\right\rangle \sigma_{H}^{n-2} \text { for all } X \in \mathfrak{X}^{1}(H) \text {. }
$$


Remark 15. We note that, in general, $\mathscr{H}_{H} \notin L_{\text {loc }}^{1}\left(S ; \sigma_{R}^{n-1}\right)$; see [Danielli et al. 2012]. However, it is always true that $\mathscr{H}_{H} \in L_{\text {loc }}^{1}\left(S ; \sigma_{H}^{n-1}\right)$; see, for instance, [Montefalcone 2012].

Remark 16. Let $S \subset \mathbb{G}$ be a hypersurface of class $C^{2}$ and $v$ the outward-pointing unit normal vector along $S$. For any $X \in \mathfrak{X}(\mathbb{G})$ let us set $X^{\perp}:=\langle X, v\rangle v$ and $X^{\top}:=X-X^{\perp}$ to denote the riemannian normal and tangential components of $X$ at any point of $S$. We would like to stress that formula (5) can be seen as a particular case of a general integral formula, the so-called first variation formula of the $H$-perimeter. More precisely, the first variation formula is given by

$$
I_{S}\left(X, \sigma_{H}^{n-1}\right)=\int_{S}\left(-\mathscr{H}_{H}\left\langle X^{\perp}, v\right\rangle+\operatorname{div}_{T S}\left(X^{\top}\left|\mathscr{P}_{H} v\right|-\left\langle X^{\perp}, v\right\rangle v_{H}^{\top}\right)\right) \sigma_{R}^{n-1},
$$

where $I_{S}\left(X, \sigma_{H}^{n-1}\right)$ denotes the first derivative of the $H$-perimeter under a smooth variation of $S$ with initial velocity $X$; see [Montefalcone 2012, Theorem 4.6]. Formula (6) also holds if $C_{S} \neq \varnothing$, but in this case we need to assume $\mathscr{H}_{H} \in$ $L_{\text {loc }}^{1}\left(S ; \sigma_{R}^{n-1}\right)$. We observe that, in the case of the first Heisenberg group $\mathbb{\boxplus}^{1}$, this formula coincides with that of Ritoré and Rosales [2008, Lemma 4.3, p. 14]. Note that, if $X=X_{H} \in \mathfrak{X}(H)$, then

$$
\begin{aligned}
X_{H}^{\top}\left|\mathscr{P}_{H} v\right|-\left\langle X_{H}^{\perp}\right. & , v\rangle v_{H}^{\top} \\
& =\left(X_{H}-\left|\mathscr{P}_{H} v\right|\left\langle X_{H}, v_{H}\right\rangle v\right)\left|\mathscr{P}_{H} v\right|-\left|\mathscr{P}_{H} v\right|\left\langle X_{H}, v\right\rangle\left(v_{H}-\left|\mathscr{P}_{H} v\right| v\right) \\
& =\left(X_{H}-\left\langle X_{H}, v\right\rangle v_{H}\right)\left|\mathscr{P}_{H} v\right|=\mathscr{P}_{H S}\left(X_{H}\right)\left|\mathscr{P}_{H} v\right|,
\end{aligned}
$$

where we have used the fact that $v=\left|\mathscr{P}_{H} v\right| v_{H}+\sum_{\alpha \in I_{V}} v_{\alpha} X_{\alpha}$ at each NC point. Finally, inserting this into (6), we obtain an equivalent form of (5). In particular, for any $X \in \mathfrak{X}(H)$ the function $\mathscr{D}_{H S} X$ turns out to be the Lie derivative of the differential $(n-1)$-form $\sigma_{H}^{n-1}\llcorner S$ with respect to the initial velocity $X$ of a smooth variation of $S$. Roughly speaking, this can be rephrased by saying that the differential $(n-1)$-form $\left(\mathscr{D}_{H S} X\right) \sigma_{H}^{n-1} \in \Lambda^{n-1}\left(T^{*} S\right)$ is the "infinitesimal" first variation of $S$.

Formula (5) holds true even if $C_{S} \neq \varnothing$, at least under suitable assumptions.

Definition 17. Let $X \in C^{1}\left(S \backslash C_{S}, H S\right)$ and set $\left.\alpha_{X}:=(X\lrcorner \sigma_{H}^{n-1}\right)\left.\right|_{S}$. We say that $X$ is admissible (for the horizontal divergence formula) if the differential forms $\alpha_{X}$ and $d \alpha_{X}$ are continuous on all of $S$, or, more generally, if $\alpha, d \alpha \in L^{\infty}(S)$ and $l_{S}^{*} \alpha \in L^{\infty}(\partial S)$. We say that $\phi \in C_{H S}^{2}\left(S \backslash C_{S}\right)$ is admissible if $\operatorname{grad}_{H S} \phi$ is admissible for the horizontal divergence formula.

We stress that, if the differential forms $\alpha_{X}$ and $d \alpha_{X}$ are continuous on all of $S$ (or, more generally, if $\alpha, d \alpha \in L^{\infty}(S)$ and $\iota_{S}^{*} \alpha \in L^{\infty}(\partial S)$, where $\imath_{S}: \partial M \rightarrow \bar{M}$ is the natural inclusion), then Stokes' formula holds true; see, for instance, [Taylor 2006]. This fact motivates the following: 
Corollary 18. Let $S \subset \mathbb{G}$ be a compact hypersurface of class $C^{2}$ with boundary $\partial S$ of class $C^{1}$. Then:

(i) $\int_{S} \mathscr{D}_{H S} X \sigma_{H}^{n-1}=\int_{\partial S}\left\langle X, \eta_{H S}\right\rangle \sigma_{H}^{n-2}$ for every admissible $X \in C^{1}\left(S \backslash C_{S}, H S\right)$.

(ii) $\int_{S} \mathscr{L}_{H S} \phi \sigma_{H}^{n-1}=\int_{\partial S}\left\langle\operatorname{grad}_{H S} \phi, \eta_{H S}\right\rangle \sigma_{H}^{n-2}$ for every admissible $\phi \in C_{H S}^{2}\left(S \backslash C_{S}\right)$.

(iii) If $\partial S=\varnothing$, then

$$
-\int_{S} \varphi \mathscr{L}_{H S} \varphi \sigma_{H}^{n-1}=\int_{S}\left|\operatorname{grad}_{H S} \varphi\right|^{2} \sigma_{H}^{n-1}
$$

for every $\varphi \in C_{H S}^{2}\left(S \backslash C_{S}\right)$ such that $\varphi^{2}$ is admissible.

The last formula holds even if $\partial S \neq \varnothing$, but for compactly supported functions. One can show that $\varphi^{2}$ is admissible if and only if $\varphi \in C_{H S}^{2}\left(S \backslash C_{S}\right) \cap W_{H S}^{1,2}\left(S, \sigma_{H}^{n-1}\right)$, where we have set $W_{H S}^{1,2}\left(S, \sigma_{H}^{n-1}\right):=\left\{\varphi \in L^{2}\left(S, \sigma_{H}^{n-1}\right):\left|\operatorname{grad}_{H S} \varphi\right| \in L^{2}\left(S, \sigma_{H}^{n-1}\right)\right\}$. We also remark that any vector field $X \in C^{1}(S, H S)$ turns out to be admissible. Analogously, any $\varphi \in C_{H S}^{2}(S)$ is admissible.

Lemma 19. Let $x_{H}:=\sum_{i \in I_{H}} x_{i} X_{i}$ be the "horizontal position vector" and let $g_{H}$ denote its component along the H-normal $v_{H}$; i.e., $g_{H}:=\left\langle x_{H}, v_{H}\right\rangle$. In the sequel, the function $g_{H}$ will be called "horizontal support function" of $x_{H}$. Then, we have:

(i) $\operatorname{div}_{H} x_{H}=h$;

(ii) $\mathscr{D}_{H S}\left(x_{H S}\right)=(h-1)+g_{H} \mathscr{H}_{H}+\left\langle C_{H} v_{H}, x_{H S}\right\rangle$ at each NC point $x \in S \backslash C_{S}$, where $x_{H S}:=x_{H}-g_{H} v_{H}$.

Proof. We have $\operatorname{div}_{H} x_{H}=\sum_{i=1}^{h}\left\langle\nabla_{X_{i}} x_{H}, X_{i}\right\rangle=\sum_{i, j=1}^{h}\left(X_{i}\left(x_{j}\right)+\left\langle\nabla_{X_{i}} X_{j}, X_{i}\right\rangle\right)=$ $\sum_{i, j=1}^{h} \delta_{i}^{j}=h$, where $\delta_{i}^{j}$ denotes Kronecker's delta; here we have used $\mathscr{f}_{H}\left(x_{H}\right)=$ $\mathbf{I d}_{h}$ and $\left\langle\nabla_{X_{i}} X_{j}, X_{i}\right\rangle=0$ for all $i, j \in I_{H}$; see Definition 6 and formula (6). Furthermore, by definition, one has $\operatorname{div}_{H S} x_{H}=\operatorname{div}_{H} x_{H}-\left\langle\nabla_{v_{H}} x_{H}, v_{H}\right\rangle$. Hence $\operatorname{div}_{H S} x_{H}=h-\left\langle v_{H}, v_{H}\right\rangle=h-1$. Furthermore, by definition, we have

$$
\operatorname{div}_{H S} x_{H S}=\sum_{i=2}^{h}\left\langle\nabla_{\tau_{i}}\left(x_{H}-g_{H} v_{H}\right), \tau_{i}\right\rangle,
$$

where we have used an orthonormal horizontal frame $\underline{\tau}_{H}:=\left\{\tau_{1}, \ldots, \tau_{h}\right\}$ in an open neighborhood $U \subset \mathbb{G}$ of $S$ such that $\tau_{1}(x)=v_{H}(x)$ at any $x \in S \backslash C_{S}$; see, for instance, Definition 3.4 in [Montefalcone 2007a]. Starting from (7), we compute $\operatorname{div}_{H S} x_{H S}=\sum_{i=2}^{h}\left(\left\langle\tau_{i}, \tau_{i}\right\rangle-g_{H}\left\langle\nabla_{\tau_{i}}^{H} v_{H}, \tau_{i}\right\rangle\right)=(h-1)-g_{H} \operatorname{div}_{H} v_{H}=(h-1)+g_{H} \mathscr{H}_{H}$ for every $x \in S \backslash C_{S}$. The thesis easily follows from the definition of $\mathscr{D}_{H S}$.

A simple consequence of Corollary 18 and Lemma 19 is given by the following: 
Corollary 20 (Minkowski-type formula). Let $S \subset \mathbb{G}$ be a compact hypersurface of class $C^{2}$. Let $x_{H}=\sum_{i \in I_{H}} x_{i} X_{i}$ be the horizontal position vector. Furthermore, set $g_{H}=\left\langle x_{H}, v_{H}\right\rangle$ and $x_{H S}=x-g_{H} v_{H}$ for every $x \in S \backslash C_{S}$. Then

$$
\int_{S}\left((h-1)+g_{H} \mathscr{H}_{H}+\left\langle C_{H} \nu_{H}, x_{H S}\right\rangle\right) \sigma_{H}^{n-1}=0 .
$$

Proof. It is enough to apply Corollary 18 to the horizontal tangent vector field $x_{H S} \in$ $C^{1}\left(S \backslash C_{S}, H S\right)$. Using Remark 15 and Lemma 19 the thesis easily follows.

Definition 21 (eigenvalue problems for $\mathscr{L}_{H S}$ ). Let $S \subset \mathbb{G}$ be a compact hypersurface of class $C^{2}$ without boundary. Then we look for solutions of class $C_{H S}^{2}\left(S \backslash C_{S}\right) \cap$ $W_{H S}^{1,2}\left(S, \sigma_{H}^{n-1}\right)$ to the problem

$$
\left(\mathrm{P}_{1}\right) \quad\left\{\begin{aligned}
-\mathscr{L}_{H S} \psi & =\lambda \psi \\
\int_{S} \psi \sigma_{H}^{n-1} & =0 .
\end{aligned}\right.
$$

If $\partial S \neq \varnothing$, we look for solutions of class $C_{H S}^{2}\left(S \backslash C_{S}\right) \cap W_{H S}^{1,2}\left(S, \sigma_{H}^{n-1}\right)$ to the problems

$$
\left(\mathrm{P}_{2}\right) \quad\left\{\begin{array} { r l } 
{ - \mathscr { L } _ { H S } \psi } & { = \lambda \psi ; } \\
{ \psi | _ { \partial S } } & { = 0 ; }
\end{array} \quad ( \mathrm { P } _ { 3 } ) \quad \left\{\begin{array}{rl}
-\mathscr{L}_{H S} \psi & =\lambda \psi ; \\
\left.\frac{\partial \psi}{\partial \eta_{H S}}\right|_{\partial S} & =0 .
\end{array}\right.\right.
$$

We explicitly remark that $\partial \psi / \partial \eta_{H S}=\left\langle\operatorname{grad}_{H S} \psi, \eta_{H S}\right\rangle$.

The problems $\left(\mathrm{P}_{1}\right),\left(\mathrm{P}_{2}\right)$ and $\left(\mathrm{P}_{3}\right)$ generalize to our context the classical closed, Dirichlet and Neumann eigenvalue problems for the Laplace-Beltrami operator on riemannian manifolds; see [Chavel 1984; 1993].

Finally, we recall a recent general result about the size of horizontal tangencies to noninvolutive distributions, which applies to our Carnot setting; see Theorem 4.5 in [Balogh et al. 2010].

Theorem 22 (generalized Derridj's theorem). Let $\mathbb{G}$ be a k-step Carnot group.

(i) If $S \subset \mathbb{G}$ is a hypersurface of class $C^{2}$, the euclidean-Hausdorff dimension of the characteristic set $C_{S}$ of $S$ satisfies $\operatorname{dim}_{\text {Еu-Наи }}\left(C_{N}\right) \leq n-2$.

(ii) If $V=H^{\perp} \subset T \mathbb{G}$ satisfies $\operatorname{dim} V \geq 2$ and $N \subset \mathbb{G}$ is an $(n-2)$-dimensional submanifold of class $C^{2}$, then the euclidean-Hausdorff dimension of the characteristic set $C_{N}$ of $N$ satisfies $\operatorname{dim}_{\text {Eu-Hau }}\left(C_{N}\right) \leq n-3$.

Remark 23. Let $N \subset \mathbb{G}$ be an $(n-2)$-dimensional submanifold of class $C^{2}$. This smoothness condition is sharp; see [Balogh et al. 2010]. Moreover, we stress that $\operatorname{dim} V=1$ just for Heisenberg groups and 2-step Carnot groups having 1dimensional center. For Heisenberg groups $\mathbb{H}^{n}, n>1$, using Frobenius' theorem yields $\operatorname{dim}_{\text {Eu-Hau }}\left(C_{N}\right) \leq n$, where $n=\frac{1}{2} \operatorname{dim} H$; see also [Balogh et al. 2010]. On the contrary, 1-dimensional curves in $\mathbb{\boxplus}^{1}$ can be horizontal or transversal to $H$. For 
2-step groups having 1-dimensional center (or, equivalently, horizontal bundle $H$ of codimension 1) a simple analysis shows that $\operatorname{dim}_{\text {Eu-Hau }}\left(C_{N}\right)=n-2$ if, and only if, $\mathbb{G}$ reduces to the direct product of $\mathbb{H}^{1}$ and of a euclidean space $\mathbb{R}^{h-2}$.

\section{Isoperimetric constants and the first eigenvalue of $\mathscr{L}_{H S}$ on compact hypersurfaces}

As a consequence of the coarea formula (4) we may generalize to the Carnot groups setting some results about isoperimetric constants and global Poincaré inequalities for which we refer the reader to [Chavel 1984; 1993]; see also [Cheeger 1970; Yau 1975].

Let $S \subset \mathbb{G}$ be a compact hypersurface of class $C^{2}$ with (or without) boundary. Similarly as in the riemannian setting (see [Cheeger 1970; Yau 1975]), we may give the following:

Definition 24. The isoperimetric constant $\operatorname{Isop}(S)$ of $S$ is defined as follows:

- If $\partial S=\varnothing$, we set

$$
\operatorname{Isop}(S):=\inf \frac{\sigma_{H}^{n-2}(N)}{\min \left\{\sigma_{H}^{n-1}\left(S_{1}\right), \sigma_{H}^{n-1}\left(S_{2}\right)\right\}},
$$

where the infimum is taken over all $C^{2}$-smooth $(n-2)$-dimensional submanifolds $N$ of $S$ which divide $S$ into two hypersurfaces $S_{1}, S_{2}$ with common boundary $N=\partial S_{1}=\partial S_{2}$.

- If $\partial S \neq \varnothing$, we set

$$
\operatorname{Isop}(S):=\inf \frac{\sigma_{H}^{n-2}(N)}{\sigma_{H}^{n-1}\left(S_{1}\right)},
$$

where $N \subset S$ is a smooth hypersurface of $S$ such that $N \cap \partial S=\varnothing$ and $S_{1}$ is the unique $C^{2}$-smooth $(n-2)$-dimensional submanifold of $S$ such that $N=\partial S_{1}$.

Here $\partial S, S_{1}, S_{2}$ and $N=\partial S_{i}(i=1,2)$ are not assumed to be connected.

This definition requires some comments. As recalled in the introduction, in the riemannian setting analogous isoperimetric constants were introduced in [Cheeger 1970], in order to give a geometric lower bound for the smallest eigenvalue of the Laplace-Beltrami operator on smooth compact riemannian manifolds. This definition was somewhat motivated by an example of Calabi, the so-called dumbbell manifold, homeomorphic to $\mathbf{S}^{2}$. Actually, an analysis of this example shows that, in order to bound $\lambda$ from below, the diameter and the volume are not enough.

We also have to recall that these isoperimetric constants turn out to be strictly positive. Although this claim turns out to be (more or less) elementary in dimension 
$n=2$, it becomes a bit more difficult when $n>2$; see [Cheeger 1970]. Some years after Cheeger's result, Yau [1975] reconsidered the isoperimetric constants and demonstrated that $\lambda$ has a bound in terms of volume, diameter and (of a lower bound of the) Ricci curvature. See the survey [Li 1982] for a glimpse on this topic.

Below we shall generalize some of the results of [Yau 1975]. Our results will follow the original scheme, which is based mainly on a suitable use of the coarea formula for smooth functions. Note also that, instead of $C^{\infty}$-smooth hypersurfaces, here we are considering hypersurfaces of class $C^{2}$. We have to observe that all the results could also be stated for $C^{1}$ hypersurfaces. But the delicate matter here is that in our setting, new difficulties come from the presence of characteristic points and, in the $C^{1}$ case, it is not simple to prove that isoperimetric constants are strictly positive. Actually, the following further hypothesis seems to be unavoidable in order to have nonzero isoperimetric constants:

(H) Every $C^{2}$-smooth $(n-2)$-dimensional submanifold $N \subset S$ satisfies

$$
\operatorname{dim} C_{N}<n-2 .
$$

This assumption can be overcome by using the generalized Derridj's theorem, (Theorem 22); see also Remark 23. As a consequence, the results of this section are "meaningful" (in the sense that the isoperimetric constants do not vanish) at least for any Carnot group $\mathbb{G}$ such that $\operatorname{dim} V \geq 2$ and for all Heisenberg groups $\llbracket^{n}$, with $n>1$.

Theorem 25. Let $S \subset \mathbb{G}$ be a compact hypersurface of class $C^{2}$.

(i) If $\partial S=\varnothing$, then

$$
\operatorname{Isop}(S)=\inf \frac{\int_{S}\left|\operatorname{grad}_{H S} \psi\right| \sigma_{H}^{n-1}}{\int_{S}|\psi| \sigma_{H}^{n-1}},
$$

where the infimum is taken over all $C^{2}$-smooth functions on $S$ such that $\int_{S} \psi \sigma_{H}^{n-1}=0$.

(ii) If $\partial S \neq \varnothing$, then

$$
\operatorname{Isop}(S)=\inf \frac{\int_{S}\left|\operatorname{grad}_{H S} \psi\right| \sigma_{H}^{n-1}}{\int_{S}|\psi| \sigma_{H}^{n-1}},
$$

where the infimum is taken over all $C^{2}$-smooth functions on $S$ such that $\left.\psi\right|_{\partial S}=0$.

Warning 26. The definition of $\operatorname{Isop}(S)$ can be weakened. For instance, part (i) of Definition 24 can be given by assuming $S$ of class $C^{1}$ and then by taking the infimum over all $(n-2)$-dimensional submanifolds $N$ of $S$ of class $C^{1}$ which divide $S$ into two hypersurfaces $S_{1}, S_{2}$ with common boundary $N=\partial S_{1}=\partial S_{2}$. 
In this case, Theorem 25(i) holds, without modifications, by taking the infimum over $C_{H S}^{1}$-smooth functions. If $\partial S \neq \varnothing$ an analogous claim holds, for the other isoperimetric constant. Furthermore, equivalent remarks can be given for all the results of this section. Nevertheless, as already said, this weaker formulation seems to be less meaningful because of the presence of characteristic points.

Warning 27. Throughout this section, we shall fix a homogeneous distance $\varrho$ on $\mathbb{G}$ of class $C^{1}$ outside the diagonal of $\mathbb{G}$.

Proof of Theorem 25. The proof repeats almost verbatim the arguments of Theorem 1 in [Yau 1975]. We just prove the theorem for $\partial S=\varnothing$ since the other case is analogous. First, let us prove the inequality

$$
\operatorname{Isop}(S) \leq \inf \frac{\int_{S}\left|\operatorname{grad}_{H S} \psi\right| \sigma_{H}^{n-1}}{\int_{S}|\psi| \sigma_{H}^{n-1}},
$$

where $\psi \in C^{2}(S)$ and $\int_{S} \psi \sigma_{H}^{n-1}=0$. To prove this inequality let us consider the auxiliary functions $\psi^{+}=\max \{0, \psi\}, \psi^{-}=\max \{0,-\psi\}$. By applying the coarea formula (4) and the definition of $\operatorname{Isop}(S)$ we get that

$$
\int_{S}\left|\operatorname{grad}_{H S} \psi^{ \pm}\right| \sigma_{H}^{n-1}=\int_{0}^{+\infty} \sigma_{H}^{n-2}\left\{x \in S: \psi^{ \pm}=t\right\} d t \geq \operatorname{Isop}(S) \int_{S}\left|\psi^{ \pm}\right| \sigma_{H}^{n-1}
$$

Now we shall prove the reversed inequality. So let us assume that $\sigma_{H}^{n-1}\left(S_{1}\right) \leq$ $\sigma_{H}^{n-1}\left(S_{2}\right)$ and let $\epsilon>0$. By making use of the fixed homogeneous distance $\varrho$ on $\mathbb{G}$, we now define a function $\psi_{\epsilon}: S \rightarrow \mathbb{R}$ by setting

$$
\begin{aligned}
& \left.\psi_{\epsilon}(x)\right|_{S_{1}}:=\left\{\begin{array}{cc}
\frac{\varrho(x, N)}{\epsilon} & \text { if } \varrho(x, N) \leq \epsilon, \\
1 & \text { if } \varrho(x, N)>\epsilon,
\end{array}\right. \\
& \left.\psi_{\epsilon}(x)\right|_{S_{2}}:=\left\{\begin{array}{cc}
-\alpha \frac{\varrho(x, N)}{\epsilon} & \text { if } \varrho(x, N) \leq \epsilon, \\
-\alpha & \text { if } \varrho(x, N)>\epsilon,
\end{array}\right.
\end{aligned}
$$

where the constant $\alpha$ depends on $\epsilon$ and is chosen in a way that $\int_{S} \psi_{\epsilon} \sigma_{H}^{n-1}=0$. Obviously

$$
\lim _{\epsilon \rightarrow 0} \alpha=\frac{\sigma_{H}^{n-1}\left(S_{1}\right)}{\sigma_{H}^{n-1}\left(S_{2}\right)} .
$$

Since

$$
\begin{aligned}
\int_{S}\left|\operatorname{grad}_{H S} \psi_{\epsilon}\right| \sigma_{H}^{n-1} & =\frac{1+\alpha}{\epsilon} \int_{N_{\epsilon}:=\{x \in S: \varrho(x, N) \leq \epsilon\}}\left|\operatorname{grad}_{H S} \varrho(x, N)\right| \sigma_{H}^{n-1} \\
& =\frac{1+\alpha}{\epsilon} \int_{0}^{\epsilon} \sigma_{H}^{n-2}\left\{x \in N_{\epsilon}: \varrho(x, N)=t\right\} d t
\end{aligned}
$$


one gets

$$
\lim _{\epsilon \rightarrow 0} \int_{S}\left|\operatorname{grad}_{H S} \psi_{\epsilon}\right| \sigma_{H}^{n-1}=(1+\alpha) \sigma_{H}^{n-2}(N) .
$$

Moreover $\lim _{\epsilon \rightarrow 0} \int_{S}\left|\psi_{\epsilon}\right| \sigma_{H}^{n-1}=\sigma_{H}^{n-1}\left(S_{1}\right)+\alpha \sigma_{H}^{n-1}\left(S_{2}\right)$. Putting it all together we get

$$
\inf _{\psi} \frac{\int_{S}\left|\operatorname{grad}_{H S} \psi\right| \sigma_{H}^{n-1}}{\int_{S}|\psi| \sigma_{H}^{n-1}} \leq \lim _{\epsilon \rightarrow 0} \frac{\int_{S}\left|\operatorname{grad}_{H S} \psi_{\epsilon}\right| \sigma_{H}^{n-1}}{\int_{S}\left|\psi_{\epsilon}\right| \sigma_{H}^{n-1}} \leq \frac{\sigma_{H}^{n-1}(N)}{\sigma_{H}^{n-2}\left(S_{1}\right)} .
$$

If we take the infimum over $N$ and $S_{1}$, the inequality follows.

Corollary 28. Let $\lambda_{1}$ be the first nonzero eigenvalue of either the closed eigenvalue problem or the Dirichlet eigenvalue problem (see Definition 21). Then we have $\lambda_{1} \geq \frac{1}{4}(\operatorname{Isop}(S))^{2}$.

Proof. We just prove the first claim, as the second claim is similar. Let $\psi$ be an eigenfunction of $\mathscr{L}_{H S}$ corresponding to $\lambda_{1}$. Then

$$
\begin{aligned}
\lambda_{1} & =-\frac{\int_{S} \psi \mathscr{L}_{H S} \psi \sigma_{H}^{n-1}}{\int_{S}|\psi|^{2} \sigma_{H}^{n-1}}=\frac{\int_{S}\left|\operatorname{grad}_{H S} \psi\right|^{2} \sigma_{H}^{n-1}}{\int_{S}|\psi|^{2} \sigma_{H}^{n-1}}=\frac{\int_{S}\left|\operatorname{grad}_{H S} \psi\right|^{2} \sigma_{H}^{n-1}}{\left(\int_{S}|\psi|^{2} \sigma_{H}^{n-1}\right)^{2}} \int_{S}|\psi|^{2} \sigma_{H}^{n-1} \\
& \geq \frac{\left(\int_{S}|\psi|\left|\operatorname{grad}_{H S} \psi\right| \sigma_{H}^{n-1}\right)^{2}}{\left(\int_{S}|\psi|^{2} \sigma_{H}^{n-1}\right)^{2}}=\frac{1}{4} \frac{\left(\int_{S}\left|\operatorname{grad}_{H S} \psi^{2}\right| \sigma_{H}^{n-1}\right)^{2}}{\left(\int_{S} \psi^{2} \sigma_{H}^{n-1}\right)^{2}} \geq \frac{(\operatorname{Isop}(S))^{2}}{4},
\end{aligned}
$$

where we have used Theorem 25 together with the Cauchy-Schwarz inequality.

We now extend, to Carnot groups, another isoperimetric constant and some related facts which, in the riemannian case, were studied in [Yau 1975].

Definition 29. The isoperimetric constant $\operatorname{Isop}_{0}(S)$ of any $C^{2}$-smooth compact hypersurface $S \subset \mathbb{G}$ with boundary $\partial S$ is given by

$$
\operatorname{Isop}_{0}(S):=\inf \left\{\frac{\sigma_{H}^{n-2}\left(\partial S_{1} \cap \partial S_{2}\right)}{\min \left\{\sigma_{H}^{n-1}\left(S_{1}\right), \sigma_{H}^{n-1}\left(S_{2}\right)\right\}}\right\},
$$

where the infimum is taken over all decompositions $S=S_{1} \cup S_{2}$ such that $\sigma_{H}^{n-1}\left(S_{1} \cap S_{2}\right)=0$.

Theorem 30. Let $S \subset \mathbb{G}$ be a compact hypersurface of class $C^{2}$ with boundary. Then

$$
\operatorname{Isop}_{0}(S)=\inf \left\{\frac{\int_{S}\left|\operatorname{grad}_{H S} \psi\right| \sigma_{H}^{n-1}}{\inf _{\beta \in \mathbb{R}} \int_{S}|\psi-\beta| \sigma_{H}^{n-1}}\right\},
$$

where the infimum is taken over all $C^{2}$-functions defined on $S$. 
Proof. The proof is analogous to that of Theorem 6 in [Yau 1975]. First, let us prove the inequality

$$
\operatorname{Isop}(S) \leq \inf \frac{\int_{S}\left|\operatorname{grad}_{H S} \psi\right| \sigma_{H}^{n-1}}{\int_{S}|\psi| \sigma_{H}^{n-1}} .
$$

To this end, let us define the functions $\psi^{+}:=\max \{0, \psi-k\}, \psi^{-}:=-\min \{0, \psi-k\}$, where $k \in \mathbb{R}$ is any constant such that

$$
\sigma_{H}^{n-1}\left\{x \in S: \psi^{+}>0\right\} \leq \frac{1}{2} \sigma_{H}^{n-1}(S), \quad \sigma_{H}^{n-1}\left\{x \in S: \psi^{-}>0\right\} \leq \frac{1}{2} \sigma_{H}^{n-1}(S) .
$$

By using again the coarea formula (4) together with the definition of $\operatorname{Isop}_{0}(S)$ we get that

$$
\int_{S}\left|\operatorname{grad}_{H S} \psi^{ \pm}\right| \sigma_{H}^{n-1}=\int_{0}^{+\infty} \sigma_{H}^{n-2}\left\{x \in S: \psi^{ \pm}=t\right\} d t \geq \operatorname{Isop}(S) \int_{S}\left|\psi^{ \pm}\right| \sigma_{H}^{n-1} .
$$

We prove the other inequality. Assuming $\sigma_{H}^{n-1}\left(S_{1}\right) \leq \sigma_{H}^{n-1}\left(S_{2}\right)$ and $\epsilon>0$, we define the function

(9) $\left.\psi_{\epsilon}(x)\right|_{S_{1}}:=1,\left.\quad \psi_{\epsilon}(x)\right|_{S_{2}}:=\left\{\begin{array}{cl}1-\frac{\varrho\left(x, \partial S_{1} \cap \partial S_{2}\right)}{\epsilon} & \left.\text { if } \varrho\left(x, \partial S_{1} \cap \partial S_{2}\right)\right) \leq \epsilon, \\ 0 & \left.\text { if } \varrho\left(x, \partial S_{1} \cap \partial S_{2}\right)\right)>\epsilon .\end{array}\right.$

Furthermore, one can find a constant $k(\epsilon)$ satisfying

$$
\int_{S}\left|\psi_{\epsilon}-k(\epsilon)\right| \sigma_{H}^{n-1}=\inf _{\beta \in \mathbb{R}} \int_{S}\left|\psi_{\epsilon}-\beta\right| \sigma_{H}^{n-1}
$$

and such that $k(\epsilon) \rightarrow 0$ for $\epsilon \rightarrow 0^{+}$. Hence

$$
\lim _{\epsilon \rightarrow 0}\left\{\frac{\int_{S}\left|\operatorname{grad}_{H S} \psi_{\epsilon}\right| \sigma_{H}^{n-1}}{\inf _{\beta \in \mathbb{R}} \int_{S}\left|\psi_{\epsilon}-\beta\right| \sigma_{H}^{n-1}}\right\} \leq \frac{\sigma_{H}^{n-2}\left(\partial S_{1} \cap \partial S_{2}\right)}{\min \left\{\sigma_{H}^{n-1}\left(S_{1}\right), \sigma_{H}^{n-1}\left(S_{2}\right)\right\}} .
$$

Corollary 31. Let $S \subset \mathbb{G}$ be a compact hypersurface of class $C^{2}$. Then

$$
\int_{S}|\psi-k|^{2} \sigma_{H}^{n-1} \leq \frac{4}{\left(\operatorname{Isop}_{0}(S)\right)^{2}} \int_{S}\left|\operatorname{grad}_{H S} \psi\right|^{2} \sigma_{H}^{n-1}
$$

for every $\psi \in C^{2}(S)$ and every $k \in \mathbb{R}$ such that

$$
\sigma_{H}^{n-1}\{x \in S: \psi \geq k\} \geq \frac{1}{2} \sigma_{H}^{n-1}(S), \quad \sigma_{H}^{n-1}\{x \in S: \psi \leq k\} \geq \frac{1}{2} \sigma_{H}^{n-1}(S) .
$$

Furthermore, if $\psi \in C^{2}(S)$ and $\int_{S} \psi \sigma_{H}^{n-1}=0$, then

$$
\int_{S}|\psi|^{2} \sigma_{H}^{n-1} \leq \frac{4}{\left(\operatorname{Isop}_{0}(S)\right)^{2}} \int_{S}\left|\operatorname{grad}_{H S} \psi\right|^{2} \sigma_{H}^{n-1} .
$$


Proof. One has $\int_{S}\left(\psi^{+} \cdot \psi^{-}\right) \sigma_{H}^{n-1}=0$, where the functions $\psi^{ \pm}$are defined as in the proof of Theorem 30. Moreover, by using once more coarea formula, we get

$$
\begin{aligned}
\int_{S}|\psi-k|^{2} \sigma_{H}^{n-1} & =\int_{S}\left|\psi^{+}+\psi^{-}\right|^{2} \sigma_{H}^{n-1} \leq \int_{S}\left|\psi^{+}\right|^{2} \sigma_{H}^{n-1}+\int_{S}\left|\psi^{-}\right|^{2} \sigma_{H}^{n-1} \\
& \leq \frac{1}{\operatorname{Isop}_{0}(S)}\left(\int_{S}\left|\operatorname{grad}_{H S}\left(\psi^{+}\right)^{2}\right| \sigma_{H}^{n-1}+\int_{S}\left|\operatorname{grad}_{H S}\left(\psi^{-}\right)^{2}\right| \sigma_{H}^{n-1}\right) \\
& \leq \frac{2}{\operatorname{Isop}_{0}(S)} \int_{S}\left(\psi^{+}+\psi^{-}\right)\left|\operatorname{grad}_{H S} \psi\right| \sigma_{H}^{n-1} \\
& \leq \frac{2}{\operatorname{Isop}_{0}(S)}\left\|\psi^{+}+\psi^{-}\right\|_{L^{2}\left(S ; \sigma_{H}^{n-1}\right)}\left\|\operatorname{grad}_{H S} \psi\right\|_{L^{2}\left(S ; \sigma_{H}^{n-1}\right)} .
\end{aligned}
$$

This proves (10). In order to prove (11) we note that the hypothesis $\int_{S} \psi \sigma_{H}^{n-1}=0$ actually implies that

$$
\int_{S} \psi^{2} \sigma_{H}^{n-1}=\inf _{k \in \mathbb{R}} \int_{S}(\psi-k)^{2} \sigma_{H}^{n-1},
$$

which, together with (10), implies the thesis of the theorem.

\section{Two upper bounds on $\lambda_{1}$}

Below we shall extend two (nowadays classical) inequalities obtained, respectively, by Chavel and Reilly in the euclidean/riemannian setting. An important feature of these results is that they give explicit upper bounds for the first nontrivial eigenvalue (of the Laplacian) of a compact submanifold of $\mathbb{R}^{n}$. For further details we refer to [Chavel 1978] and [Reilly 1977]; see also [Heintze 1988]. To begin with, let $\Omega \subsetneq \mathbb{G}$ be a bounded domain and assume that $S:=\partial \Omega$ is a connected hypersurface of class $C^{2}$, with orientation given by the outward normal vector $v$. Moreover, let $x_{H}$ be the horizontal position vector field and let us apply the usual divergence formula. We also set $\sigma_{R}^{n}=\mathscr{V}_{0}{ }^{n}$. We have

$$
h \mathscr{V o l}^{n}(\Omega)=\int_{\Omega} \operatorname{div}_{H} x_{H} \sigma_{R}^{n}=\int_{\partial \Omega}\left\langle x_{H}, v\right\rangle \sigma_{R}^{n-1}=\int_{S}\left\langle x_{H}, v_{H}\right\rangle \sigma_{H}^{n-1},
$$

where we have used Lemma 19(i). Furthermore, we may further assume that the "center of mass" of $\partial \Omega$ (with respect to the $H$-perimeter) is placed at the identity $0 \in \mathbb{G}$. In other words, let us assume that $\int_{S} x_{i} \sigma_{H}^{n-1}=0$ for every $i \in I_{H}=\{1, \ldots, h\}$, where $x_{H} \equiv\left(x_{1}, \ldots, x_{i}, \ldots, x_{h}\right)$ is the horizontal position vector; see Lemma 19.

The last assumption is justified by the following:

Lemma 32. Let $S \subset \mathbb{G}$ be a compact hypersurface of class $C^{i}(i \geq 1)$. We can always choose a system of exponential coordinates $x=\exp \left(x_{1}, \ldots, x_{n}\right)$ on $\mathbb{G}$ such that $\int_{S} x_{i} \sigma_{H}^{n-1}(x)=0$ for every $i \in I_{H}=\{1, \ldots, h\}$. 
Proof. Let

$$
a_{i}:=\frac{\int_{S} x_{i} \sigma_{H}^{n-1}(x)}{\sigma_{H}^{n-1}(S)} \quad \text { for all } i \in I_{H}=\{1, \ldots, h\}
$$

and $a_{H} \equiv\left(a_{1}, \ldots, a_{i}, \ldots, a_{h}\right)$. Set $a:=\exp \left(a_{H}, 0_{V}\right)$, where the symbol $0_{V}$ denotes the origin of $V \subset \mathfrak{g}$. Consider the change of variables $y:=\Phi(x)=a^{-1} \bullet x(x \in \mathbb{G})$. Equivalently, we have $\Phi(x)=L_{a^{-1}}(x)$, where $L_{a^{-1}}$ is the left-translation by $a^{-1}=-a$; see Section 1.1. The usual change of variables formula together with standard properties of the pull-back imply the following chain of equalities:

$$
\begin{aligned}
\int_{\Phi(S)} f(y) \sigma_{H}^{n-1}(y) & =\int_{S} f(\Phi(x)) \operatorname{gac}(\Phi)(x) \sigma_{H}^{n-1}(x) \\
& =\int_{S} \Phi^{*}\left(f \sigma_{H}^{n-1}\right)=\int_{S}(f \circ \Phi)\left(\Phi^{*} \sigma_{H}^{n-1}\right)
\end{aligned}
$$

for every smooth function $f: S \rightarrow \mathbb{R}$; see, for instance, [Lee 2003, Lemma 9.11, p. 214]. Using the left-invariance of the $H$-perimeter yields $\operatorname{fac}(\Phi)=1$, or equivalently, $\Phi^{*} \sigma_{H}^{n-1}=\sigma_{H}^{n-1}$. Now, let us assume that $f(y):=y_{i}$ for any $i \in I_{H}$. Equivalently, let $f$ be the $i$-th exponential coordinate of the variable $y \in \mathbb{G}$. Note also that $(f \circ \Phi)(x)=\Phi_{i}(x)=-a_{i}+x_{i}$ for any $i \in I_{H}$. Actually, this follows from the fact that the group law $\bullet$ acts linearly on the horizontal layer; see (1). Then, using (12) yields

$$
\int_{\Phi(S)} y_{i} \sigma_{H}^{n-1}(y)=\int_{S}\left(-a_{i}+x_{i}\right) \sigma_{H}^{n-1}(x)=0 \quad \text { for all } i \in I_{H},
$$

which achieves the proof.

We therefore get that

$$
\begin{aligned}
h \mathscr{V o l}^{n}(\Omega) & =\int_{S}\left\langle x_{H}, v_{H}\right\rangle \sigma_{H}^{n-1} \leq \int_{S}\left|x_{H}\right| \sigma_{H}^{n-1} \leq \sqrt{\sigma_{H}^{n-1}(S)} \sqrt{\int_{S}\left|x_{H}\right|^{2} \sigma_{H}^{n-1}} \\
& =\sqrt{\sigma_{H}^{n-1}(S)} \sqrt{\int_{S} \sum_{i \in I_{H}} x_{i}^{2} \sigma_{H}^{n-1}} \leq \sqrt{\frac{\sigma_{H}^{n-1}(S)}{\lambda_{1}}} \sqrt{\int_{S} \sum_{i \in I_{H}}\left|\operatorname{grad}_{H S} x_{i}\right|^{2} \sigma_{H}^{n-1}},
\end{aligned}
$$

where the last identity follows from Lord Rayleigh's characterization of the first nontrivial eigenvalue $\lambda_{1}$ of the operator $\mathscr{L}_{H S}$ on $S$. Now a direct computation gives the pointwise identity $\sum_{i \in I_{H}}\left|\operatorname{grad}_{H S} x_{i}\right|^{2}=h-1$. Hence, putting it all together, we have shown the following:

Theorem 33. Let $\Omega \subsetneq \mathbb{G}$ be a bounded domain with $C^{2}$ boundary $S=\partial D$. Let $\lambda_{1}$ be the first (nontrivial) eigenvalue of the operator $\mathscr{L}_{H S}$ on $S$. Then

$$
\sqrt{\lambda_{1}} \frac{\mathscr{V o l}^{n}(\Omega)}{\sigma_{H}^{n-1}(S)} \leq \frac{\sqrt{h-1}}{h} .
$$


We now discuss another geometric inequality, which looks very similar to the last one. More precisely, let $S$ be a $C^{2}$-smooth compact hypersurface without boundary. So let us make use of Rayleigh's principle:

$$
\lambda_{1} \int_{S} \varphi^{2} \sigma_{H}^{n-1} \leq \int_{S}\left|\operatorname{grad}_{H S} \varphi\right|^{2} \sigma_{H}^{n-1}
$$

for any function $\varphi \in C^{2}\left(S \backslash C_{S}\right) \cap W^{1,2}{ }_{H S}\left(S, \sigma_{H}^{n-1}\right)$ satisfying $\int_{S} \varphi \sigma_{H}^{n-1}=0$. Again, we assume that the center of mass of $S=\partial \Omega$ is placed at $0 \in \mathbb{G}$ so that $\int_{S} x_{i} \sigma_{H}^{n-1}=0$ for every $i \in I_{H}$. Hence, similarly as above, we get that $\lambda_{1} \int_{S}\left|x_{H}\right|^{2} \sigma_{H}^{n-1}=\lambda_{1} \sum_{i \in I_{H}} \int_{S} x_{i}^{2} \sigma_{H}^{n-1} \leq \lambda_{1} \sum_{i \in I_{H}} \int_{S}\left|\operatorname{grad}_{H S} x_{i}\right|^{2} \sigma_{H}^{n-1}=(h-1) \sigma_{H}^{n-1}(S)$. At this point, we reformulate Corollary 20 as follows:

$$
\int_{S}\left((h-1)+\left\langle\left(\mathscr{H}_{H} v_{H}+C_{H} v_{H}\right), x_{H}\right\rangle\right) \sigma_{H}^{n-1}=0 .
$$

From this identity and the Cauchy-Schwarz inequality, we easily get that

$$
\begin{aligned}
(h-1) \sigma_{H}^{n-1}(S) & \leq \sqrt{\int_{S}\left|x_{H}\right|^{2} \sigma_{H}^{n-1}} \sqrt{\int_{S}\left|\mathscr{H}_{H} v_{H}+C_{H} v_{H}\right|^{2} \sigma_{H}^{n-1}} \\
& \leq \sqrt{\int_{S}\left|x_{H}\right|^{2} \sigma_{H}^{n-1}} \sqrt{\int_{S}\left(\mathscr{H}_{H}^{2}+\left|C_{H} v_{H}\right|^{2}\right) \sigma_{H}^{n-1}} .
\end{aligned}
$$

Therefore

$$
\frac{\left((h-1) \sigma_{H}^{n-1}(S)\right)^{2}}{\int_{S}\left(\mathscr{H}_{H}^{2}+\left|C_{H} v_{H}\right|^{2}\right) \sigma_{H}^{n-1}} \leq \int_{S}\left|x_{H}\right|^{2} \sigma_{H}^{n-1}
$$

and hence

$$
\lambda_{1} \frac{\left((h-1) \sigma_{H}^{n-1}(S)\right)^{2}}{\int_{S}\left(\mathscr{H}_{H}^{2}+\left|C_{H} v_{H}\right|^{2}\right) \sigma_{H}^{n-1}} \leq(h-1) \sigma_{H}^{n-1}(S),
$$

which proves the following:

Theorem 34. Let $\Omega \subsetneq \mathbb{G}$ be a bounded domain with $C^{2}$ boundary $S=\partial D$ and $v$ the outward-pointing unit normal vector along $S$. Let $\lambda_{1}$ be the first eigenvalue of the operator $\mathscr{L}_{H S}$ on $S$. The following upper bound for $\lambda_{1}$ holds:

$$
\lambda_{1} \leq \frac{\int_{S}\left(\mathscr{H}_{H}^{2}+\left|C_{H} v_{H}\right|^{2}\right) \sigma_{H}^{n-1}}{(h-1) \sigma_{H}^{n-1}(S)}=\frac{f_{S}\left(\mathscr{H}_{H}^{2}+\left|C_{H} v_{H}\right|^{2}\right) \sigma_{H}^{n-1}}{h-1} .
$$




\section{Horizontal linear isoperimetric inequalities}

Let $S \subset \mathbb{G}$ be a compact hypersurface of class $C^{2}$ with (or without) boundary. Let $x_{H}$ be the horizontal position vector of $S$ and set $x_{H S}:=x_{H}-g_{H} v_{H}$ where $g_{H}=\left\langle x_{H}, v_{H}\right\rangle$ is the horizontal support function of $S$; see Lemma 19. We recall that

$$
\int_{S}\left((h-1)+g_{H} \mathscr{H}_{H}+\left\langle C_{H} v_{H}, x_{H S}\right\rangle\right) \sigma_{H}^{n-1}=\int_{\partial S}\left\langle x_{H}, \eta_{H S}\right\rangle \sigma_{H}^{n-2} ;
$$

see Corollary 20. Note that, if $\partial S=\varnothing$, then the boundary integral vanishes. From this we easily get that

$$
(h-1) \sigma_{H}^{n-1}(S) \leq \int_{S}\left(\left|g_{H}\right|\left|\mathscr{H}_{H}\right|+\left|\left\langle C_{H} v_{H}, x_{H S}\right\rangle\right|\right) \sigma_{H}^{n-1}+\int_{\partial S}\left|\left\langle x_{H}, \eta_{H S}\right\rangle\right| \sigma_{H}^{n-2} .
$$

Remark 35 (assumptions on $\varrho$ ). Let $\varrho(x)=\varrho(0, x)=\|x\|_{\varrho}$ be a homogeneous norm on $\mathbb{G}$ and let $\varrho(x, y)=\left\|y^{-1} \cdot x\right\|_{\varrho}$ be the associated (homogeneous) distance on $\mathbb{G}$. In this section we assume the following:

(i) $\varrho$ is piecewise $C^{1}$ outside the diagonal of $\mathbb{G}$;

(ii) $\left|\operatorname{grad}_{H} \varrho\right| \leq 1$ at each regular point of $\varrho$;

(iii) $\left|x_{H}\right| \leq \varrho(x, 0)$ for all $x \in \mathbb{G}$.

Example 36. On the Heisenberg group $\mathbb{U}^{n}$, the CC-distance $d_{\mathrm{CC}}$ satisfies these assumptions. Another example is the distance associated with the Koranyi norm defined by $\|x\|_{\varrho}:=\varrho(x)=\sqrt[4]{\left|x_{H}\right|^{4}+16 t^{2}}$ for $x=\exp \left(x_{H}, t\right) \in \mathbb{Q}^{n}$. This norm is homogeneous and $C^{\infty}$-smooth out of $0 \in \mathbb{Q}^{n}$ and satisfies conditions (ii) and (iii). This example can easily be generalized to any Carnot group having step 2 and satisfying $C_{H}^{\alpha} C_{H_{2}}^{\beta}=-\mathbf{1}_{H_{i}} \delta_{\alpha}^{\beta},\left(\alpha, \beta \in I_{H_{2}}\right)$. Actually, in this case, one can show that the homogeneous norm $\|\cdot\|_{\varrho}$, defined by $\|x\|_{\varrho}:=\sqrt[4]{\left|x_{H}\right|^{4}+16\left|x_{H_{2}}\right|^{2}}$ for all $x=\exp \left(x_{H}, x_{H_{2}}\right)$ satisfies all the conditions in Remark 35 .

Let $R$ be the radius of the $\varrho$-ball $B_{\varrho}(0, R)$, centered at the identity 0 of the group $\mathbb{G}$ and circumscribed about $S$. It is important to remark that, because of the left-invariance of the $H$-perimeter, we may replace 0 with any $x \in \mathbb{G}$. Below, we shall estimate (by the Cauchy-Schwarz inequality) the right-hand side of (14). To this aim, note that $g_{H} \leq\left|x_{H}\right| \leq\|x\|_{\varrho}$. So we have

$$
(h-1) \sigma_{H}^{n-1}(S) \leq R\left(\int_{S}\left(\left|\mathscr{H}_{H}\right|+\left|C_{H} v_{H}\right|\right) \sigma_{H}^{n-1}+\sigma_{H}^{n-2}(\partial S)\right),
$$

which is a linear inequality. Obviously, if $S$ is $H$-minimal, i.e., $\mathscr{H}_{H}=0$, we have

$$
(h-1) \sigma_{H}^{n-1}(S) \leq R\left(\int_{S}\left|C_{H} v_{H}\right| \sigma_{H}^{n-1}+\sigma_{H}^{n-2}(\partial S)\right) .
$$


Furthermore, if $\mathscr{H}_{H}^{0}:=\max \left\{\mathscr{H}_{H}(x) \mid x \in S\right\}$, one gets

$$
\sigma_{H}^{n-1}(S)\left((h-1)-R \mathscr{H}_{H}^{0}\right) \leq R\left(\int_{S}\left|C_{H} \nu_{H}\right| \sigma_{H}^{n-1}+\sigma_{H}^{n-2}(\partial S)\right) .
$$

Equivalently, we have

$$
R \geq \frac{(h-1) \sigma_{H}^{n-1}(S)}{\mathscr{H}_{H}^{0} \sigma_{H}^{n-1}(S)+\left(\int_{S}\left|C_{H} v_{H}\right| \sigma_{H}^{n-1}+\sigma_{H}^{n-2}(\partial S)\right)},
$$

and, by assuming $R \mathscr{H}_{H}^{0}<h-1$, we also get

$$
\sigma_{H}^{n-1}(S) \leq \frac{R\left(\int_{S}\left|C_{H} \nu_{H}\right| \sigma_{H}^{n-1}+\sigma_{H}^{n-2}(\partial S)\right)}{(h-1)-R \mathscr{H}_{H}^{0}} .
$$

Here, we just remark that there are no closed compact $H$-minimal hypersurfaces immersed in Carnot groups. This fact can be proved by using the first variation formula of the $H$-perimeter; see [Montefalcone 2012]. The previous formulae have been proved for hypersurfaces with boundary, but they hold even if $\partial S=\varnothing$. More precisely:

Proposition 37. Let $S \subset \mathbb{G}$ be a compact hypersurface of class $C^{2}$ without boundary. Let $R$ be the radius of the $\varrho$-ball $B_{\varrho}(0, R)$, centered at the identity 0 of the group $\mathbb{G}$ and circumscribed about $S$. Then

$$
\begin{aligned}
(h-1) \sigma_{H}^{n-1}(S) & \leq R \int_{U}\left(\left|\mathscr{H}_{H}\right|+\left|C_{H} v_{H}\right|\right) \sigma_{H}^{n-1} ; \\
R & \geq \frac{(h-1) \sigma_{H}^{n-1}(S)}{\mathscr{H}_{H}^{0} \sigma_{H}^{n-1}(S)+\int_{S}\left|C_{H} v_{H}\right| \sigma_{H}^{n-1}} ; \\
\sigma_{H}^{n-1}(S) & \leq \frac{R \int_{S}\left|C_{H} v_{H}\right| \sigma_{H}^{n-1}}{(h-1)-R \mathscr{H}_{H}^{0}} .
\end{aligned}
$$

4.1. Application: a weak monotonicity formula. In the sequel, we shall set $S_{t}=$ $S \cap B_{\varrho}(x, t)$. The "natural" monotonicity formula which can be deduced from the inequality (15) is contained in:

Proposition 38. The following inequality holds for $\mathscr{L}^{1}$-a.e. $t>0$ :

$$
-\frac{d}{d t} \frac{\sigma_{H}^{n-1}\left(S_{t}\right)}{t^{h-1}} \leq \frac{1}{t^{h-1}}\left(\int_{S_{t}}\left(\left|\mathscr{H}_{H}\right|+\left|C_{H} v_{H}\right|\right) \sigma_{H}^{n-1}+\sigma_{H}^{n-2}\left(\partial S \cap B_{\varrho}(x, t)\right)\right) .
$$

Proof. Since we are assuming that the homogeneous distance $\varrho$ is smooth (at least piecewise $C^{1}$ ), by applying the classical Sard's theorem we get that $S_{t}$ is a $C^{2}$-smooth manifold with boundary for $\mathscr{L}^{1}$-a.e. $t>0$ (or, equivalently, this claim 
follows by intersecting $S$ with the boundary of a $\varrho$-ball $B_{\varrho}(x, t)$ centered at $x$ and of radius $t$ ). So let us apply formula (13) for the set $S_{t}$. We have

$$
(h-1) \sigma_{H}^{n-1}\left(S_{t}\right) \leq t\left(\int_{S_{t}}\left(\left|\mathscr{H}_{H}\right|+\left|C_{H} v_{H}\right|\right) \sigma_{H}^{n-1}+\sigma_{H}^{n-2}\left(\partial S_{t}\right)\right),
$$

where $t$ is the radius of a $\varrho$-ball centered at $x$ and intersecting $S$. Since

$$
\partial S_{t}=\left\{\partial S \cap B_{\varrho}(x, t)\right\} \cup\left\{\partial B_{\varrho}(x, t) \cap S\right\},
$$

we get

$$
\begin{aligned}
(h-1) \sigma_{H}^{n-1}\left(S_{t}\right) \leq t(\underbrace{\int_{S_{t}}\left(\left|\mathscr{H}_{H}\right|+\left|C_{H} v_{H}\right|\right) \sigma_{H}^{n-1}}_{=: \mathscr{A}(t)} & +\underbrace{\sigma_{H}^{n-2}\left(\partial S \cap B_{\varrho}(x, t)\right)}_{=: \mathscr{B}(t)} \\
& \left.+\sigma_{H}^{n-2}\left(\partial B_{\varrho}(x, t) \cap S\right)\right) .
\end{aligned}
$$

Now let us consider the function $\psi(y):=\left\|y^{-1} \bullet x\right\|_{\varrho}$ for all $y \in S$. By hypothesis, $\psi$ is a $C^{1}$-smooth function - at least piecewise - satisfying $\left|\operatorname{grad}_{H} \psi\right| \leq 1$; see Remark 35. So we may apply the coarea formula to this function. Since $\left|\operatorname{grad}_{H S} \psi\right| \leq$ $\left|\operatorname{grad}_{H} \psi\right|$, we easily get that

$$
\begin{aligned}
\sigma_{H}^{n-1}\left(S_{t_{1}}\right)-\sigma_{H}^{n-1}\left(S_{t}\right) & \geq \int_{S_{t_{1} \backslash S_{t}}}\left|\operatorname{grad}_{H S} \psi\right| \sigma_{H}^{n-1}=\int_{t}^{t_{1}} \sigma_{H}^{n-2}\left\{\psi^{-1}[s] \cap S\right\} d s \\
& =\int_{t}^{t_{1}} \sigma_{H}^{n-2}\left(\partial B_{\varrho}(x, s) \cap S\right) d s .
\end{aligned}
$$

From the last inequality we infer that

$$
\frac{d}{d t} \sigma_{H}^{n-1}\left(S_{t}\right) \geq \sigma_{H}^{n-2}\left(\partial B_{\varrho}(x, t) \cap S\right)
$$

for $\mathscr{L}^{1}$-a.e. $t>0$. Hence, from this inequality and (24), we obtain

$$
(h-1) \sigma_{H}^{n-1}\left(S_{t}\right) \leq t\left(\mathscr{A}(t)+\mathscr{B}(t)+\frac{d}{d t} \sigma_{H}^{n-1}\left(S_{t}\right)\right),
$$

which is an equivalent form of (23).

We have to notice however that, in order to prove an "intrinsic" isoperimetric inequality, the number $(h-1)$ in the previous differential inequality is not the correct one, which is $(Q-1)$. This fact motivates a further study, made by the author in [Montefalcone 2009; 2010]. 


\section{A theorem about nonhorizontal graphs in 2-step Carnot groups}

We begin by describing our result in the simpler setting of the first Heisenberg group $\mathbb{W}^{1}$; see also [Montefalcone 2007b]. For the notation, see Example 7.

Theorem 39 (Heinz's estimate for $T$-graphs). Let $S=\left\{p=\exp (x, y, t) \in \mathbb{U}^{1}\right.$ : $t=f(x, y)$ for all $\left.(x, y) \in \mathbb{R}^{2}\right\}$ be a $T$-graph of class $C^{2}$ over the $x y$-plane. If $\left|\mathscr{H}_{H}\right| \geq C>0$, then

$$
C \mathscr{H}_{\mathrm{Eu}}^{2}\left(\mathscr{P}_{x y}(\mathcal{U})\right) \leq \mathscr{H}_{\mathrm{Eu}}^{1}\left(\mathscr{P}_{x y}(\partial \mathcal{U})\right)
$$

for every $C^{1}$-smooth relatively compact open set $u \subset S$. Hence, taking $U:=$ $S \cap C_{r}(\mathscr{T})$, where $C_{r}(\mathscr{T})$ denotes a vertical cylinder of radius $r$ around the $T$-axis $\mathscr{T}:=\left\{p=\exp (0,0, t) \in \mathbb{H}^{1}, t \in \mathbb{R}\right\}$, yields, for every $r>0$,

$$
r \leq \frac{2}{C}
$$

It follows that any entire $x y$-graph of class $C^{2}$ having constant (or just bounded) horizontal mean curvature $\mathscr{H}_{H}$ must be necessarily a $H$-minimal surface. To see this fact, it is enough to send $r \rightarrow+\infty$. The proof of the previous theorem is elementary. More precisely, one uses the following identity:

$$
\left.-\int_{U} \mathscr{H}_{H} \varpi \sigma_{H}^{2}=\int_{\partial \widetilde{U}} v_{H}\right\lrcorner d \theta,
$$

where $\theta=T^{*}=d t+\frac{y d x-x d y}{2}$ denotes the dual 1-form to the vertical direction $T$. We also have to remark that $\varpi \sigma_{H}^{2}=-d \theta=d x \wedge d y$. The previous theorem is a generalization to our context of a classical result obtained in [Heinz 1955]. This was generalized in [Chern 1965] and then by other authors in a number of different directions.

Below, we shall restrict ourselves to consider only 2-step Carnot groups.

Definition 40 (nonhorizontal graphs in 2-step Carnot groups). Let $\mathbb{G}$ be a 2-step Carnot group and let $Z=\sum_{\alpha \in I_{V}} z_{\alpha} X_{\alpha} \in V$ be a constant vertical vector. In this case, for the sake of simplicity, we reorder the variables in $\mathfrak{g}$ as $x \equiv\left(x_{Z \perp}, x_{Z}\right)$, where $x_{Z}:=\langle x, Z\rangle \in \mathbb{R}$ and $x_{Z^{\perp}}:=x-x_{Z} Z \in Z^{\perp}$. Then, we say that $S \subset \mathbb{G}$ is a $Z$-graph (over the hyperplane $Z^{\perp}$ ) if there exists a function $\psi: Z^{\perp} \rightarrow \mathbb{R}$ such that $S=\left\{p=\exp \left(x_{Z^{\perp}}, \psi\left(x_{Z^{\perp}}\right)\right) \in \mathbb{G}, x_{Z^{\perp}} \in Z^{\perp}\right\}$.

Let us fix a constant vertical vector $Z \in V$ and let $S=\left\{p=\exp \left(x_{Z^{\perp}}, \psi\left(x_{Z^{\perp}}\right)\right) \in\right.$ $\mathbb{G}, x_{Z \perp} \in Z^{\perp}$ \} be a $Z$-graph of class $C^{2}$ over the $Z^{\perp}$-hyperplane. For the sake of simplicity and without loss of generality, we may take $Z=X_{\alpha}$ for a fixed index $\alpha \in I_{V}=\{h+1, \ldots, n\}$. 
Now let us define a differential $(n-2)$-form on $S \subset \mathbb{G}$ by setting

$$
\left.\left.\xi^{\alpha}:=\left(v_{H}\right\lrcorner X_{\alpha}\right\lrcorner \sigma_{R}^{n}\right)\left.\right|_{S \backslash C_{S}} \in \Lambda^{2}\left(T^{*} S\right) .
$$

This differential $(n-2)$-form $\xi^{\alpha}$ is well-defined out of $C_{S}$ and we have to compute its exterior derivative. Below we will briefly sketch a proof, which can also be found in [Montefalcone 2007a]; see Claim 3.22.

Lemma 41. At each NC point,

$$
\left.d \xi^{\alpha}\right|_{S \backslash C_{S}}=-\left.\mathscr{H}_{H} \varpi_{\alpha} \sigma_{H}^{n-1}\right|_{S \backslash C_{S}} .
$$

Proof. Let us set $\left.\left.\zeta_{j}:=\left(X_{\alpha}\right\lrcorner X_{j}\right\lrcorner \sigma_{R}^{n}\right)\left.\right|_{S}$ for any $\alpha \in I_{V}$ and $j \in I_{H}$ and compute $\left.\left.d \zeta_{j}:=d\left(X_{\alpha}\right\lrcorner X_{j}\right\lrcorner \sigma_{R}^{n}\right)\left.\right|_{S}$. Let $\mathbb{G}$ be a $k$-step Carnot group. We claim that

$$
\left.\left.d \zeta_{j}\right|_{S \backslash C_{S}}=\sum_{k=\alpha+1}^{n} C_{\alpha j}^{k}\left(X_{k}\right\lrcorner \sigma_{R}^{n}\right)\left.\right|_{S \backslash C_{S}}=\left.\sum_{k=\alpha+1}^{n} C_{\alpha j}^{k} v_{k} \sigma_{R}^{n-1}\right|_{S \backslash C_{S}} .
$$

The proof of this claim is just a long, but elementary, calculation. Since we are assuming that $\mathbb{G}$ has step 2, using the properties of the Carnot structural constants yields $C_{\alpha j}^{k}=0$ whenever $j, k \in I_{H}$ and $\alpha \in I_{V}$. Hence $d \zeta_{j}=0$ for every $j \in I_{H}$. By linearity $\xi^{\alpha}=-\sum_{j \in I_{H}} v_{H}^{j} \zeta_{j}$, where $v_{H}^{j}=\left\langle v_{H}, X_{j}\right\rangle$ for any $j \in I_{H}$. It follows easily that $d \xi^{\alpha}=-\mathscr{H}_{H} \varpi_{\alpha} \sigma_{H}^{n-1}$, as wished.

Theorem 42 (Heinz's estimate for nonhorizontal graphs in 2-step Carnot groups). Let $\mathbb{G}$ be a 2-step Carnot group and let $Z \in V$ be a constant vertical vector. Furthermore, let $S$ be a $Z$-graph of class $C^{2}$ over the $Z^{\perp}$-hyperplane. If $\left|\mathscr{H}_{H}\right| \geq$ $C>0$, then

$$
C \mathscr{H}_{\mathrm{Eu}}^{n-1}\left(\mathscr{P}_{Z \perp}(\mathcal{U})\right) \leq \mathscr{H}_{\mathrm{Eu}}^{n-2}\left(\mathscr{P}_{Z^{\perp}}(\partial \mathcal{U})\right)
$$

for every $C^{1}$-smooth relatively compact open set $u \subset S$. Hence, taking $u:=$ $S \cap C_{r}(\mathscr{L})$, where $C_{r}(\mathscr{L})$ denotes a euclidean cylinder of radius $r$ around the $Z$-axis given by $\mathscr{E}:=\left\{p=\exp \left(0_{Z^{\perp}}, t\right) \in \mathbb{G}, t \in \mathbb{R}\right\}$, yields, for every $r>0$,

$$
r \leq \frac{n-1}{C} \text {. }
$$

Proof. Without loss of generality, we may assume $-\mathscr{H}_{H} \geq C>0$ and take $Z=X_{\alpha}$ for some fixed index $\alpha \in I_{V}$. In this case, one has

$$
\left.\left.\varpi_{\alpha} \sigma_{H}^{n-1}\right|_{S}=\left.v_{\alpha} \sigma_{R}^{n-1}\right|_{S}=\left(X_{\alpha}\right\lrcorner \sigma_{R}^{n}\right)\left.\right|_{S}=d \mathscr{H}_{\mathrm{Eu}}^{n-1}\left\llcorner X_{\alpha}^{\perp},\right.
$$

where the last identity follows from our assumption that $S$ is a $X_{\alpha}$-graph. By using Lemma 41 and Stokes' formula, we obtain the integral identity

$$
\left.\left.-\int_{U} \mathscr{H}_{H} \varpi_{\alpha} \sigma_{H}^{n-1}=\int_{\partial \vartheta} v_{H}\right\lrcorner X_{\alpha}\right\lrcorner \sigma_{R}^{n} .
$$


Furthermore, we have

$$
\begin{aligned}
-\int_{\mathcal{U}} \mathscr{H}_{H} \varpi_{\alpha} \sigma_{H}^{n-1} & =-\int_{\mathscr{P}_{X}{ }_{\alpha}(\vartheta)} \mathscr{H}_{H} d \mathscr{H}_{\mathrm{Eu}}^{n-1}, \\
\left.\int\left(v_{H}\right\lrcorner d \mathscr{H}_{\mathrm{Eu}}^{n-1}\right)\left.\right|_{\mathscr{P}_{X}{ }_{\alpha}(\partial \vartheta)} & =\int\left\langle v_{H}, \eta\right\rangle d \mathscr{H}_{\mathrm{Eu}}^{n-2}\left\llcorner\mathscr{P}_{X_{\alpha}^{\perp}(\partial \vartheta)} .\right.
\end{aligned}
$$

Putting it all together, we get $C \mathscr{H}_{\mathrm{Eu}}^{n-1}\left(\mathscr{P}_{X_{\alpha}^{\perp}}(\mathcal{U})\right) \leq \mathscr{H}_{\mathrm{Eu}}^{n-2}\left(\mathscr{P}_{X_{\alpha}^{\perp}}(\partial U)\right)$, which proves (26) when $Z=X_{\alpha}$. The thesis follows by linearity. Finally, (27) follows from (26) and the elementary calculation

$$
\frac{\mathscr{H}_{\mathrm{Eu}}^{n-2}\left(\partial B_{\mathrm{Eu}}^{n-1}\right)}{\mathcal{H}_{\mathrm{Eu}}^{n-1}\left(B_{\mathrm{Eu}}^{n-1}\right)}=n-1,
$$

where $B_{\mathrm{Eu}}^{n-1}$ denotes a euclidean unit ball in $Z^{\perp} \cong \mathbb{R}^{n-1}$.

It follows that an entire $Z$-graph of class $C^{2}$ over the $Z^{\perp}$-hyperplane having constant (or bounded) horizontal mean curvature $\mathscr{H}_{H}$ must be necessarily a $H$ minimal hypersurface.

\section{Local Poincaré-type inequality}

By using an elementary technique, somehow analogous to the one used in Section 4, we will state a local Poincaré-type inequality for smooth compactly supported functions on NC domains. First we need the following:

Definition 43. Let $S \subset \mathbb{G}$ be a hypersurface of class $C^{2}$ and let $U \subseteq S$ be an open domain. We say that $U$ is uniformly noncharacteristic (abbreviated UNC) if

$$
\sup _{x \in \mathscr{U}}|\varpi(x)|=\sup _{x \in \mathscr{U}} \frac{\left|\mathscr{P}_{V} v(x)\right|}{\left|\mathscr{P}_{H} v(x)\right|}<+\infty .
$$

We stress that

$$
\left|C_{H} v_{H}\right|=\left|\sum_{\alpha \in I_{V}} \omega_{\alpha} C_{H}^{\alpha} v_{H}\right| \leq \sum_{\alpha \in I_{V}}\left|\omega_{\alpha}\right|\left\|C_{H}^{\alpha}\right\|_{\mathrm{Gr}} \leq \frac{C}{\left|\mathscr{P}_{H} \nu\right|},
$$

where $C:=\sum_{\alpha \in I_{V}}\left\|C_{H}^{\alpha}\right\|_{\text {Gr }}$ only depends on the structural constants of $\mathfrak{g}$. Set

$$
R_{u}:=\frac{1}{2\left[\left\|\mathscr{H}_{H}\right\|_{L^{\infty}(U)}+C\|\varpi\|_{L^{\infty}(U)}\right]} .
$$

From (28), we have $\left|C_{H} v_{H}\right| \leq C \max _{\alpha \in I_{V}}\left|\varpi_{\alpha}\right|$. Moreover, $\int_{B}\left|\varpi_{\alpha}\right| \sigma_{H}^{n-1}=$ $\int_{B}\left|v_{\alpha}\right| \sigma_{R}^{n-1} \leq \sigma_{R}^{n-1}(B)$ for every Borel set $B \subseteq S$.

Theorem 44. Let $S \subset \mathbb{G}$ be a hypersurface of class $C^{2}$. Let $U \subset S$ be a uniformly $N C$ open domain. Then, for all $x \in \mathcal{U}$ and for all $R \leq \min \left\{\operatorname{dist}_{\varrho}(x, \partial U), R_{u}\right\}$, 


$$
\left(\int_{U_{R}}|\psi|^{p} \sigma_{H}^{n-1}\right)^{\frac{1}{p}} \leq C_{p} R\left(\int_{U_{R}}\left|\operatorname{grad}_{H S} \psi\right|^{p} \sigma_{H}^{n-1}\right)^{\frac{1}{p}}, \quad p \in[1,+\infty[,
$$

for every $\psi \in C_{H S}^{1}\left(\cup_{R}\right) \cap C_{0}\left(u_{R}\right)$. More generally, let $\tilde{u} \subset \cup$ be a bounded open subset of $U$ with smooth boundary such that $\operatorname{diam}_{\varrho}(\tilde{u}) \leq 2 \min \left\{\operatorname{dist}_{\varrho}(x, \partial u), R_{\varkappa}\right\}$.

Then

(30) $\left(\int_{\tilde{\widetilde{U}}}|\psi|^{p} \sigma_{H}^{n-1}\right)^{\frac{1}{p}} \leq C_{p} \operatorname{diam}_{\varrho}(\tilde{\mathcal{U}})\left(\int_{\widetilde{\widetilde{U}}}\left|\operatorname{grad}_{H S} \psi\right|^{p} \sigma_{H}^{n-1}\right)^{\frac{1}{p}}, \quad p \in[1,+\infty[$,

for every $\psi \in C_{H S}^{1}(\widetilde{u}) \cap C_{0}(\tilde{u})$.

In this theorem one can take $C_{p}:=\frac{2 p}{2 h-3}$.

Proof. Let us set $\psi_{\varepsilon}:=\sqrt{\varepsilon^{2}+\psi^{2}}(\varepsilon \geq 0)$. By applying Theorem 14 with $X=\psi_{\varepsilon} x_{H}$ we get

$$
\begin{aligned}
\int_{U_{R}}\left\{\psi_{\varepsilon}\left((h-1)+g_{H} \mathscr{H}_{H}+\left\langle C_{H} v_{H}, x_{H S}\right\rangle\right)+\left\langle\operatorname{grad}_{H S} \psi_{\varepsilon}, x_{H}\right\rangle\right\} \sigma_{H}^{n-1} & \\
& =\int_{\partial U_{R}} \psi_{\varepsilon}\left\langle x_{H}, \eta_{H S}\right\rangle \sigma_{H}^{n-2},
\end{aligned}
$$

and so

$$
\begin{aligned}
(h-1) \int_{U_{R}} \psi_{\varepsilon} \sigma_{H}^{n-1} \leq & R\left(\int_{U_{R}}\left[\psi_{\varepsilon}\left(\left|\mathscr{H}_{H}\right|+\left|C_{H} v_{H}\right|\right)+\left|\operatorname{grad}_{H S} \psi_{\varepsilon}\right|\right] \sigma_{H}^{n-1}+\int_{\partial \cup_{R}} \psi_{\varepsilon} \sigma_{H}^{n-2}\right) \\
\leq R\left(\left\|\mathscr{H}_{H}\right\|_{L} \infty\left(U_{R}\right)\right. & \left.+C\|\varpi\|_{L^{\infty}\left(u_{R}\right)}\right) \int_{U_{R}} \psi_{\varepsilon} \sigma_{H}^{n-1} \\
& +R\left(\int_{U_{R}}\left|\operatorname{grad}_{H S} \psi_{\varepsilon}\right| \sigma_{H}^{n-1}+\int_{\partial U_{R}} \psi_{\varepsilon} \sigma_{H}^{n-2}\right) .
\end{aligned}
$$

By using Fatou's lemma and the estimate $R \leq R_{\varkappa}$ we get that

$$
\begin{aligned}
(h- & 1) \int_{U_{R}}|\psi| \sigma_{H}^{n-1} \\
& \leq(h-1) \liminf _{\varepsilon \rightarrow 0^{+}} \int_{\bigcup_{R}} \psi_{\varepsilon} \sigma_{H}^{n-1} \\
& \leq \frac{1}{2} \lim _{\varepsilon \rightarrow 0^{+}} \int_{U_{R}} \psi_{\varepsilon} \sigma_{H}^{n-1}+R \lim _{\varepsilon \rightarrow 0^{+}}\left(\int_{U_{R}}\left|\operatorname{grad}_{H S} \psi_{\varepsilon}\right| \sigma_{H}^{n-1}+\int_{\partial U_{R}} \psi_{\varepsilon} \sigma_{H}^{n-2}\right) .
\end{aligned}
$$

Obviously, $\psi_{\varepsilon} \rightarrow|\psi|$ and $\left|\operatorname{grad}_{H S} \psi_{\varepsilon}\right| \rightarrow\left|\operatorname{grad}_{H S} \psi\right|$ as long as $\varepsilon \rightarrow 0$; moreover $|\psi|=0$ along $\partial u_{R}$. Now since, as it is well-known, $\left|\operatorname{grad}_{H S}\right| \psi|| \leq\left|\operatorname{grad}_{H S} \psi\right|$, we easily get the claim by Lebesgue's dominated convergence theorem. So we have shown that

$$
\int_{U_{R}}|\psi| \sigma_{H}^{n-1} \leq \frac{2 R}{2 h-3} \int_{U_{R}}\left|\operatorname{grad}_{H S} \psi\right| \sigma_{H}^{n-1}
$$


for every $\psi \in C_{H S}^{1}\left(u_{R}\right) \cap C_{0}\left(u_{R}\right)$. Finally, the general case follows by Hölder's inequality. More precisely, let us use the last inequality with $|\psi|$ replaced by $|\psi|^{p}$. This implies

$$
\begin{aligned}
\int_{U_{R}}|\psi|^{p} \sigma_{H}^{n-1} & \leq \frac{2 R}{(2 h-3)} \int_{U_{R}} p|\psi|^{p-1}\left|\operatorname{grad}_{H S} \psi\right| \sigma_{H}^{n-1} \\
& \leq \frac{2 p R}{(2 h-3)}\left(\int_{U_{R}}|\psi|^{(p-1) q} \sigma_{H}^{n-1}\right)^{\frac{1}{q}}\left(\int_{U_{R}}\left|\operatorname{grad}_{H S} \psi\right|^{p} \sigma_{H}^{n-1}\right)^{\frac{1}{p}},
\end{aligned}
$$

where $\frac{1}{p}+\frac{1}{q}=1$. This achieves the proof of (29). Finally, (30) can be proved by repeating the same arguments as above, just by replacing $R$ with diam( $(\widetilde{U})$.

With some extra hypotheses one can show that (29) still holds up to the characteristic set.

Theorem 45. Let $S \subset \mathbb{G}$ be a hypersurface of class $C^{2}$ with (or without) boundary $\partial S$. We assume that $S$ has bounded horizontal mean curvature $\mathcal{H}_{H}$ and that $\operatorname{dim} C_{S}<n-2$. Furthermore, let $u_{\epsilon}(\epsilon>0)$ be a family of open subsets of $S$ with $C^{1}$ boundaries, such that:

(i) $C_{S} \subset \cup_{\epsilon}$ for every $\epsilon>0$;

(ii) $\sigma_{R}^{n-1}\left(u_{\epsilon}\right) \rightarrow 0$ for $\epsilon \rightarrow 0^{+}$;

(iii) $\int_{\mathscr{U}_{\epsilon}}\left|\mathscr{P}_{H} \nu\right| \sigma_{R}^{n-2} \rightarrow 0$ for $\epsilon \rightarrow 0^{+}$.

Then, for every $x \in S$ and every (small enough) $\epsilon>0$ there exists $R_{0}:=R_{0}(x, \epsilon) \leq$ $\operatorname{dist}_{\varrho}(x, \partial S)$ such that

$$
\left(\int_{S_{R}}|\psi|^{p} \sigma_{H}^{n-1}\right)^{\frac{1}{p}} \leq C_{p} R\left(\int_{S_{R}}\left|\operatorname{grad}_{H S} \psi\right|^{p} \sigma_{H}^{n-1}\right)^{\frac{1}{p}}, \quad p \in[1,+\infty[,
$$

holds for every $\psi \in C_{H S}^{1}\left(S_{R}\right) \cap C_{0}\left(S_{R}\right)$ and every $R \leq R_{0}$, where

$$
R_{0}:=\min \left\{\operatorname{dist}_{\varrho}(x, \partial S), \frac{1}{2\left[C\left(1+\|\varpi\|_{L^{\infty}\left(S_{R} \backslash \vartheta_{\epsilon}\right)}\right)+\left\|\mathscr{H}_{H}\right\|_{L^{\infty}\left(S_{R}\right)}\right]}\right\} .
$$

Proof. Set $\psi_{\varepsilon}:=\sqrt{\varepsilon^{2}+\psi^{2}}(0 \leq \varepsilon<1)$. We shall prove the theorem for $p=1$. The general case will follow by using Hölder's inequality. Let $u_{\epsilon}(\epsilon>0)$ be as above. Fix $\epsilon_{0}>0$. For every $\epsilon \leq \epsilon_{0}$ one has

$$
\int_{U_{\epsilon}} \psi_{\varepsilon}\left|C_{H} v_{H}\right| \sigma_{H}^{n-1} \leq 2 C\|\psi\|_{L}^{\infty}\left(\cup_{\epsilon_{0}}\right) \sigma_{R}^{n-1}\left(u_{\epsilon}\right),
$$

where we have put $C:=\sum_{\alpha \in I_{V}}\left\|C_{H}^{\alpha}\right\|_{\text {Gr. }}$. Furthermore (ii) implies that for every $\delta>0$ there exists $\epsilon_{\delta}>0$ such that $\sigma_{R}^{n-1}\left(u_{\epsilon}\right)<\delta$ whenever $\epsilon<\epsilon \delta$. Taking 


$$
\begin{aligned}
& \tilde{\delta} \leq \frac{\int_{S_{R}} \psi_{\varepsilon} \sigma_{H}^{n-1}}{2\|\psi\|_{L} \infty\left(\cup_{\epsilon_{0}}\right)} \text {, one gets } \\
& \qquad \int_{U_{\epsilon}} \psi_{\varepsilon}\left|C_{H} v_{H}\right| \sigma_{H}^{n-1} \leq C \int_{S_{R}} \psi_{\varepsilon} \sigma_{H}^{n-1}
\end{aligned}
$$

for every $\epsilon \leq \min \left\{\epsilon_{\tilde{\delta}}, \epsilon_{0}\right\}$. Moreover, for any $\left.\epsilon \in\right] 0, \min \left\{\epsilon_{\tilde{\delta}}, \epsilon_{0}\right\}[$, one has

$$
\int_{S_{R} \backslash u_{\epsilon}} \psi_{\varepsilon}\left|C_{H} v_{H}\right| \sigma_{H}^{n-1} \leq C\|\varpi\|_{L}^{\infty}\left(S_{R} \backslash u_{\epsilon}\right) \int_{S_{R}} \psi_{\varepsilon} \sigma_{H}^{n-1}
$$

It follows that

$$
\int_{S_{R}} \psi_{\varepsilon}\left|C_{H} v_{H}\right| \sigma_{H}^{n-1} \leq C\left(1+\|\varpi\|_{L^{\infty}\left(S_{R} \backslash u_{\epsilon}\right)}\right) \int_{S_{R}} \psi_{\varepsilon} \sigma_{H}^{n-1} .
$$

Since, by hypothesis, the horizontal mean curvature is bounded, we clearly have

$$
\int_{S_{R}} \psi_{\varepsilon}\left|\mathscr{H}_{H}\right| \sigma_{H}^{n-1} \leq\left\|\mathscr{H}_{H}\right\|_{L^{\infty}\left(S_{R}\right)} \int_{S_{R}} \psi_{\varepsilon} \sigma_{H}^{n-1} .
$$

Applying Theorem 14 with $X=\psi_{\varepsilon} x_{H}$ (and arguing as in the proof of Theorem 44) yields

$$
\begin{array}{r}
(h-1) \int_{S_{R}} \psi_{\varepsilon} \sigma_{H}^{n-1} \leq R\left(\int_{S_{R}}\left\{\psi_{\varepsilon}\left(\left|\mathscr{H}_{H}\right|+\left|C_{H} v_{H}\right|\right)+\left|\operatorname{grad}_{H S} \psi_{\varepsilon}\right|\right\} \sigma_{H}^{n-1}+\int_{\partial S_{R}} \psi_{\varepsilon} \sigma_{H}^{n-2}\right) \\
\leq R\left[C\left(1+\|\varpi\|_{L^{\infty}\left(S_{R} \backslash U_{\epsilon}\right)}\right)+\left\|\mathscr{H}_{H}\right\|_{\left.L^{\infty}\left(S_{R}\right)\right]} \int_{S_{R}} \psi_{\varepsilon} \sigma_{H}^{n-1}\right. \\
+R\left(\int_{S_{R}}\left|\operatorname{grad}_{H S} \psi_{\varepsilon}\right| \sigma_{H}^{n-1}+\int_{\partial S_{R}} \psi_{\varepsilon} \sigma_{H}^{n-2}\right) .
\end{array}
$$

So if $R \leq R_{0}$, one gets

$$
\int_{S_{R}} \psi_{\varepsilon} \sigma_{H}^{n-1} \leq \frac{2 R}{2 h-3}\left(\int_{S_{R}}\left|\operatorname{grad}_{H S} \psi_{\varepsilon}\right| \sigma_{H}^{n-1}+\int_{\partial S_{R}} \psi_{\varepsilon} \sigma_{H}^{n-2}\right) .
$$

We have $\psi_{\varepsilon} \rightarrow|\psi|$ and $\left|\operatorname{grad}_{H S} \psi_{\varepsilon}\right| \rightarrow\left|\operatorname{grad}_{H S} \psi\right|$ as long as $\varepsilon \rightarrow 0$ and $|\psi|=0$ along $\partial S_{R}$. Since $\left|\operatorname{grad}_{H S}\right| \psi|| \leq\left|\operatorname{grad}_{H S} \psi\right|$, the thesis follows from Fatou's lemma and Lebesgue's dominated convergence theorem.

6.1. A Caccioppoli-type inequality. Our final result is a generalization of the classical Caccioppoli inequality (see, for instance, [Ambrosio 1997]) for the operator $\mathscr{L}_{H S}$ on smooth hypersurfaces.

Let $S \subset \mathbb{G}$ be a hypersurface of class $C^{2}$ and set $S_{R}:=S \cap B_{\varrho}(x, R)$ for any $x \in \mathbb{G}$. We are going to consider the functions satisfying, in the distributional sense,

$$
-\mathscr{L}_{H S} \phi=\psi \quad \text { on } S_{R},
$$

whenever $\psi \in L^{2}\left(S_{R}, \sigma_{H}^{n-1}\right)$. 
So let us take a function $\zeta \in C_{H S}^{1}\left(S_{R}\right) \cap C_{0}\left(S_{R}\right)$ such that $0 \leq \zeta \leq 1, \zeta=1$ on $S_{R / 2}=S \cap B_{\varrho}(0, R / 2)$ and $\left|\operatorname{grad}_{H S} \zeta\right| \leq C_{0} / R$. Inserting into the above equation the function $\varphi=\zeta^{2}\left(\phi-\phi_{0}\right)$, where $\phi_{0} \in \mathbb{R}$ is a fixed constant, and then integrating over $S_{R}$, yields

$$
\begin{array}{r}
\underbrace{\int_{S_{R}} \zeta^{2}\left|\operatorname{grad}_{H S} \phi\right|^{2} \sigma_{H}^{n-1}}_{=: I_{1}}+\underbrace{2 \int_{S_{R}} \zeta\left(\phi-\phi_{0}\right)\left\langle\operatorname{grad}_{H S} \zeta, \operatorname{grad}_{H S} \phi\right\rangle \sigma_{H}^{n-1}}_{=: I_{2}} \\
=\underbrace{\int_{S_{R}} \psi \zeta^{2}\left(\phi-\phi_{0}\right) \sigma_{H}^{n-1}}_{=: I_{3}}
\end{array}
$$

We have

$$
I_{2} \leq \frac{1}{2} \int_{S_{R}}|\zeta|^{2}\left|\operatorname{grad}_{H S} \phi\right|^{2} \sigma_{H}^{n-1}+\underbrace{2 \int_{S_{R}}\left|\phi-\phi_{0}\right|^{2}\left|\operatorname{grad}_{H S} \phi\right|^{2} \sigma_{H}^{n-1}}_{=: I_{4}} .
$$

Moreover $I_{4} \leq 2 C_{0}^{2} / R^{2}\left\|\phi-\phi_{0}\right\|_{L^{2}\left(S_{R}\right)}$. Now let us estimate the third integral $I_{3}$ :

$$
\begin{aligned}
\int_{S_{R}} \psi \zeta^{2}\left(\phi-\phi_{0}\right) \sigma_{H}^{n-1} & =\int_{S_{R}} 2\left((2 R \psi) \frac{\zeta^{2}\left(\phi-\phi_{0}\right)}{4 R}\right) \sigma_{H}^{n-1} \\
& \leq 4 R^{2} \int_{S_{R}} \psi^{2} \sigma_{H}^{n-1}+\frac{1}{16 R^{2}} \int_{S_{R}} \zeta^{4}\left|\phi-\phi_{0}\right|^{2} \sigma_{H}^{n-1} \\
& \leq 4 R^{2} \int_{S_{R}} 2 \psi^{2} \sigma_{H}^{n-1}+\frac{1}{R^{2}} \int_{S_{R}}\left|\phi-\phi_{0}\right|^{2} \sigma_{H}^{n-1}
\end{aligned}
$$

Since $\zeta=1$ on $S_{R / 2}$, using the previous estimates yields

$$
\int_{S_{R / 2}}\left|\operatorname{grad}_{H S} \phi\right|^{2} \sigma_{H}^{n-1} \leq \frac{2 C_{0}^{2}+1}{R^{2}} \int_{S_{R}}\left|\phi-\phi_{0}\right|^{2} \sigma_{H}^{n-1}+4 R^{2} \int_{S_{R}} \psi^{2} \sigma_{H}^{n-1} .
$$

We summarize these calculations, as follows:

Theorem 46. Let $S \subset \mathbb{G}$ be a hypersurface of class $C^{2}$; let $\phi_{0} \in \mathbb{R}$ and let $\phi$ be a distributional solution to the equation $-\mathscr{L}_{H S} \phi=\psi$ on $S_{R}$, where $\psi \in L^{2}\left(S_{R}, \sigma_{H}^{n-1}\right)$. Then, there exists a positive constant $C>0$ such that the following "Caccioppolitype" inequality holds:

$$
\int_{S_{R / 2}}\left|\operatorname{grad}_{H S} \phi\right|^{2} \sigma_{H}^{n-1} \leq C\left(\frac{1}{R^{2}} \int_{S_{R}}\left|\phi-\phi_{0}\right|^{2} \sigma_{H}^{n-1}+R^{2} \int_{S_{R}} \psi^{2} \sigma_{H}^{n-1}\right)
$$

for every (small enough) $R>0$, where $S_{R}:=S \cap B_{\varrho}(x, R)$, for any $x \in S$. 


\section{Acknowledgements}

The authors wish to thank the anonymous referee for helpful comments that improved the paper.

\section{References}

[Ambrosio 1997] L. Ambrosio, Corso introduttivo alla teoria geometrica della misura ed alle superfici minime, Scuola Normale Superiore, Pisa, 1997. MR 2000k:49001 Zbl 0977.49028

[Ambrosio 2001] L. Ambrosio, "Some fine properties of sets of finite perimeter in Ahlfors regular metric measure spaces", Adv. Math. 159:1 (2001), 51-67. MR 2002b:31002 Zbl 1002.28004

[Ambrosio and Kirchheim 2000a] L. Ambrosio and B. Kirchheim, "Currents in metric spaces", Acta Math. 185:1 (2000), 1-80. MR 2001k:49095 Zbl 0984.49025

[Ambrosio and Kirchheim 2000b] L. Ambrosio and B. Kirchheim, "Rectifiable sets in metric and Banach spaces", Math. Ann. 318:3 (2000), 527-555. MR 2003a:28009 Zbl 0966.28002

[Ambrosio et al. 2006] L. Ambrosio, F. Serra Cassano, and D. Vittone, "Intrinsic regular hypersurfaces in Heisenberg groups", J. Geom. Anal. 16:2 (2006), 187-232. MR 2007g:49072 Zbl 1085.49045

[Balogh 2003] Z. M. Balogh, "Size of characteristic sets and functions with prescribed gradient", $J$. Reine Angew. Math. 564 (2003), 63-83. MR 2005d:43007 Zbl 1051.53024

[Balogh et al. 2009] Z. M. Balogh, J. T. Tyson, and B. Warhurst, "Sub-Riemannian vs. Euclidean dimension comparison and fractal geometry on Carnot groups", Adv. Math. 220:2 (2009), 560-619. MR 2009k:53064 Zbl 1155.22011

[Balogh et al. 2010] Z. M. Balogh, C. Pintea, and H. Rohner, "Size of tangencies to non-involutive distributions", preprint, 2010, http://org.uib.no/hcaa/BaloghPinteaRohner.pdf. Zbl 06137664

[Burago and Zalgaller 1988] Y. D. Burago and V. A. Zalgaller, Geometric inequalities, Grundlehren Math. Wiss. 285, Springer, Berlin, 1988. MR 89b:52020 Zbl 0633.53002

[Capogna et al. 1994] L. Capogna, D. Danielli, and N. Garofalo, "The geometric Sobolev embedding for vector fields and the isoperimetric inequality", Comm. Anal. Geom. 2:2 (1994), 203-215. MR 96d:46032 Zbl 0864.46018

[Capogna et al. 2010] L. Capogna, G. Citti, and M. Manfredini, "Smoothness of Lipschitz minimal intrinsic graphs in Heisenberg groups $\mathbb{T}^{n}, n>1$ ”, J. Reine Angew. Math. 648 (2010), 75-110. MR 2012g:35367 Zbl 1217.53060

[Chavel 1978] I. Chavel, "On A. Hurwitz' method in isoperimetric inequalities", Proc. Amer. Math. Soc. 71:2 (1978), 275-279. MR 58 \#12845 Zbl 0395.52007

[Chavel 1984] I. Chavel, Eigenvalues in Riemannian geometry, Pure and Applied Mathematics 115, Academic Press, Orlando, FL, 1984. MR 86g:58140 Zbl 0551.53001

[Chavel 1993] I. Chavel, Riemannian geometry: A modern introduction, Cambridge Tracts in Mathematics 108, Cambridge University Press, 1993. MR 95j:53001 Zbl 0810.53001

[Chavel 2001] I. Chavel, Isoperimetric inequalities: Differential geometric and analytic perspectives, Cambridge Tracts in Mathematics 145, Cambridge University Press, 2001. MR 2002h:58040 Zbl 0988.51019

[Cheeger 1970] J. Cheeger, "A lower bound for the smallest eigenvalue of the Laplacian", pp. 195199 in Problems in analysis: A symposium in honor of Salomon Bochner (Princeton, NJ, 1969), edited by R. C. Gunning, Princeton University Press, 1970. MR 53 \#6645 Zbl 0212.44903

[Cheeger and Kleiner 2010] J. Cheeger and B. Kleiner, "Differentiating maps into $L^{1}$, and the geometry of BV functions", Ann. of Math. (2) 171:2 (2010), 1347-1385. MR 2012a:30132 Zbl 1194.22009 
[Cheng et al. 2005] J.-H. Cheng, J.-F. Hwang, A. Malchiodi, and P. Yang, "Minimal surfaces in pseudohermitian geometry”, Ann. Sc. Norm. Super. Pisa Cl. Sci. (5) 4:1 (2005), 129-177. MR 2006f:53008 Zbl 1158.53306

[Chern 1965] S.-S. Chern, "On the curvatures of a piece of hypersurface in euclidean space", $A b h$. Math. Sem. Univ. Hamburg 29 (1965), 77-91. MR 32 \#6376 Zbl 0147.20901

[Corwin and Greenleaf 1990] L. J. Corwin and F. P. Greenleaf, Representations of nilpotent Lie groups and their applications, I: Basic theory and examples, Cambridge Studies in Advanced Mathematics 18, Cambridge University Press, 1990. MR 92b:22007 Zbl 0704.22007

[Danielli et al. 2007] D. Danielli, N. Garofalo, and D. M. Nhieu, "Sub-Riemannian calculus on hypersurfaces in Carnot groups”, Adv. Math. 215:1 (2007), 292-378. MR 2009h:53061 Zbl 1129.53017

[Danielli et al. 2010] D. Danielli, N. Garofalo, and D. M. Nhieu, "Sub-Riemannian calculus and monotonicity of the perimeter for graphical strips", Math. Z. 265:3 (2010), 617-637. MR 2011e:53033 Zbl 1206.53034

[Danielli et al. 2012] D. Danielli, N. Garofalo, and D. M. Nhieu, "Integrability of the sub-Riemannian mean curvature of surfaces in the Heisenberg group", Proc. Amer. Math. Soc. 140:3 (2012), 811-821. MR 2012m:53060 Zbl 06024089

[David and Semmes 1997] G. David and S. Semmes, Fractured fractals and broken dreams: Selfsimilar geometry through metric and measure, Oxford Lecture Ser. Math. Appl. 7, Oxford University Press, New York, 1997. MR 99h:28018 Zbl 0887.54001

[Federer 1969] H. Federer, Geometric measure theory, Die Grundlehren der mathematischen Wissenschaften 153, Springer, New York, NY, 1969. MR 41 \#1976 Zbl 0176.00801

[Franchi et al. 2001] B. Franchi, R. Serapioni, and F. Serra Cassano, "Rectifiability and perimeter in the Heisenberg group", Math. Ann. 321:3 (2001), 479-531. MR 2003g:49062 Zbl 1057.49032

[Franchi et al. 2003a] B. Franchi, R. Serapioni, and F. Serra Cassano, "On the structure of finite perimeter sets in step 2 Carnot groups”, J. Geom. Anal. 13:3 (2003), 421-466. MR 2004i:49085 Zbl 1064.49033

[Franchi et al. 2003b] B. Franchi, R. Serapioni, and F. Serra Cassano, "Regular hypersurfaces, intrinsic perimeter and implicit function theorem in Carnot groups", Comm. Anal. Geom. 11:5 (2003), 909-944. MR 2004m:28008 Zbl 1077.22008

[Franchi et al. 2007] B. Franchi, R. Serapioni, and F. Serra Cassano, "Regular submanifolds, graphs and area formula in Heisenberg groups", Adv. Math. 211:1 (2007), 152-203. MR 2008h:49030 Zbl 1125.28002

[Garofalo and Nhieu 1996] N. Garofalo and D.-M. Nhieu, "Isoperimetric and Sobolev inequalities for Carnot-Carathéodory spaces and the existence of minimal surfaces", Comm. Pure Appl. Math. 49:10 (1996), 1081-1144. MR 97i:58032 Zbl 0880.35032

[Ge 1992] Z. Ge, "Betti numbers, characteristic classes and sub-Riemannian geometry", Illinois J. Math. 36:3 (1992), 372-403. MR 94f:53038 Zbl 0769.53014

[Gromov 1996] M. Gromov, “Carnot-Carathéodory spaces seen from within”, pp. 79-323 in SubRiemannian geometry, edited by A. Bellaïche and J.-J. Risler, Progr. Math. 144, Birkhäuser, Basel, 1996. MR 2000f:53034 Zbl 0864.53025

[Heintze 1988] E. Heintze, "Extrinsic upper bounds for $\lambda_{1}$ ”, Math. Ann. 280:3 (1988), 389-402. MR 89f:53091 Zbl 0628.53044

[Heinz 1955] E. Heinz, "Über Flächen mit eindeutiger Projektion auf eine Ebene, deren Krümmungen durch Ungleichungen eingeschränkt sind”, Math. Ann. 129 (1955), 451-454. MR 17,189a Zbl 0065.3720 
[Hladky and Pauls 2008] R. K. Hladky and S. D. Pauls, "Constant mean curvature surfaces in sub-Riemannian geometry”, J. Differential Geom. 79:1 (2008), 111-139. MR 2009m:53070 Zbl 1156.53038

[Koiller et al. 2001] J. Koiller, P. R. Rodrigues, and P. Pitanga, "Non-holonomic connections following Élie Cartan”, An. Acad. Brasil. Ciênc. 73:2 (2001), 165-190. MR 2002i:37097 Zbl 0999.70016

[Lee 2003] J. M. Lee, Introduction to smooth manifolds, Graduate Texts in Mathematics 218, Springer, New York, 2003. MR 2003k:58001 Zbl 1030.53001

[Li 1982] P. Li, "Poincaré inequalities on Riemannian manifolds", pp. 73-83 in Seminar on differential geometry, edited by S. T. Yau, Annals of Mathematics Studies 102, Princeton University Press, 1982. Zbl 0492.5303

[Magnani 2002] V. Magnani, Elements of geometric measure theory on sub-Riemannian groups, Scuola Normale Superiore, Pisa, 2002. MR 2005m:28002 Zbl 1064.28007

[Magnani and Vittone 2008] V. Magnani and D. Vittone, "An intrinsic measure for submanifolds in stratified groups”, J. Reine Angew. Math. 619 (2008), 203-232. MR 2009g:53046 Zbl 1146.53022

[Milnor 1976] J. Milnor, "Curvatures of left invariant metrics on Lie groups", Advances in Math. 21:3 (1976), 293-329. MR 54 \#12970 Zbl 0341.53030

[Montefalcone 2005] F. Montefalcone, "Some relations among volume, intrinsic perimeter and onedimensional restrictions of BV functions in Carnot groups", Ann. Sc. Norm. Super. Pisa Cl. Sci. (5) 4:1 (2005), 79-128. MR 2006e:49088 Zbl 1150.49022

[Montefalcone 2007a] F. Montefalcone, "Hypersurfaces and variational formulas in sub-Riemannian Carnot groups", J. Math. Pures Appl. (9) 87:5 (2007), 453-494. MR 2008d:53035 Zbl 1123.49035

[Montefalcone 2007b] F. Montefalcone, "Ipersuperfici e formule variazionali in gruppi di Carnot subRiemanniani”, lecture notes of Bruno Pini Mathematical Analysis Seminar, Università di Bologna, 2007, http://www.dm.unibo.it/seminario-pini/pdf/SemPiniMont.pdf.

[Montefalcone 2009] F. Montefalcone, "Isoperimetric, Sobolev and Poincaré inequalities on hypersurfaces in sub-Riemannian Carnot groups", preprint, 2009. arXiv 0910.5656

[Montefalcone 2010] F. Montefalcone, "Isoperimetric and Sobolev inequalities on hypersurfaces in sub-Riemannian Carnot groups”, preprint, 2010. arXiv 1012.2442

[Montefalcone 2012] F. Montefalcone, "Stable $H$-minimal hypersurfaces", 2012. arXiv 1203.5972

[Montgomery 2002] R. Montgomery, A tour of subriemannian geometries, their geodesics and applications, Mathematical Surveys and Monographs 91, American Mathematical Society, Providence, RI, 2002. MR 2002m:53045 Zbl 1044.53022

[Pansu 1982] P. Pansu, Gèometrie du group d'Heisenberg, Ph.D. thesis, Université Paris Diderot, 1982.

[Pansu 1989] P. Pansu, "Métriques de Carnot-Carathéodory et quasiisométries des espaces symétriques de rang un", Ann. of Math. (2) 129:1 (1989), 1-60. MR 90e:53058 Zbl 0678.53042

[Pansu 2005] P. Pansu, "Submanifolds and differential forms in Carnot manifolds, after M. Gromov et M. Rumin", Université Paris-Sud, 2005, http://www.math.u-psud.fr/ pansu/trento_pansu.pdf.

[Reilly 1977] R. C. Reilly, "On the first eigenvalue of the Laplacian for compact submanifolds of Euclidean space”, Comment. Math. Helv. 52:4 (1977), 525-533. MR 58 \#2657 Zbl 0382.53038

[Ritoré and Rosales 2008] M. Ritoré and C. Rosales, "Area-stationary surfaces in the Heisenberg group $\mathbb{Q}^{1}$ ”, Adv. Math. 219:2 (2008), 633-671. MR 2009h:49075 Zbl 1158.53022

[Stein 1993] E. M. Stein, Harmonic analysis: real-variable methods, orthogonality, and oscillatory integrals, Princeton Mathematical Series 43, Princeton University Press, 1993. MR 95c:42002 Zbl 0821.42001 
[Strichartz 1986] R. S. Strichartz, "Sub-Riemannian geometry", J. Differential Geom. 24:2 (1986), 221-263. Correction in 30:2 (1989), 595-596. MR 88b:53055 Zbl 0609.53021

[Taylor 2006] M. E. Taylor, Measure theory and integration, Graduate Studies in Mathematics 76, American Mathematical Society, Providence, RI, 2006. MR 2007g:28002 Zbl 1139.28001

[Varadarajan 1974] V. S. Varadarajan, Lie groups, Lie algebras, and their representations, PrenticeHall, Englewood Cliffs, NJ, 1974. MR 51 \#13113 Zbl 0371.22001

[Varopoulos et al. 1992] N. T. Varopoulos, L. Saloff-Coste, and T. Coulhon, Analysis and geometry on groups, Cambridge Tracts in Mathematics 100, Cambridge University Press, 1992. MR 95f:43008 Zbl 0813.22003

[Vershik and Gershkovich 1994] A. M. Vershik and V. Y. Gershkovich, "Nonholonomic dynamical systems: Geometry of distributions and variational problems", pp. 1-81 in Dynamical systems, vol. 7, edited by S. P. Novikov, Encycl. Math. Sci. 16, Springer, Berlin, 1994. Zbl 0797.58007

[Yau 1975] S. T. Yau, "Isoperimetric constants and the first eigenvalue of a compact Riemannian manifold”, Ann. Sci. École Norm. Sup. (4) 8:4 (1975), 487-507. MR 53 \#1478 Zbl 0325.53039

Received March 28, 2012. Revised October 1, 2012.

Francescopaolo Montefalcone

DiPARTIMENTO Di MATEMATICA

UNIVERSITÀ DEGLI STUdi Di PADOVA

VIA TRIESTE, 63

35121 PADOVA

ITALY

montefal@math.unipd.it 


\title{
PACIFIC JOURNAL OF MATHEMATICS
}

\author{
msp.org/pjm
}

Founded in 1951 by E. F. Beckenbach (1906-1982) and F. Wolf (1904-1989)

\section{EDITORS}

V. S. Varadarajan (Managing Editor)

Department of Mathematics

University of California

Los Angeles, CA 90095-1555

pacific@math.ucla.edu

Paul Balmer

Department of Mathematics

University of California

Los Angeles, CA 90095-1555

balmer@math.ucla.edu

Daryl Cooper

Department of Mathematics

University of California

Santa Barbara, CA 93106-3080 cooper@math.ucsb.edu

Jiang-Hua $\mathrm{Lu}$

Department of Mathematics

The University of Hong Kong

Pokfulam Rd., Hong Kong jhlu@maths.hku.hk
Don Blasius

Department of Mathematics University of California

Los Angeles, CA 90095-1555

blasius@math.ucla.edu

Robert Finn

Department of Mathematics Stanford University

Stanford, CA 94305-2125

finn@math.stanford.edu

Sorin Popa

Department of Mathematics

University of California

Los Angeles, CA 90095-1555

popa@math.ucla.edu

Paul Yang

Department of Mathematics

Princeton University

Princeton NJ 08544-1000

yang@math.princeton.edu

\section{PRODUCTION}

Silvio Levy, Scientific Editor, production@msp.org

\section{SUPPORTING INSTITUTIONS}

ACADEMIA SINICA, TAIPEI

CALIFORNIA INST. OF TECHNOLOGY

INST. DE MATEMÁTICA PURA E APLICADA

KEIO UNIVERSITY

MATH. SCIENCES RESEARCH INSTITUTE

NEW MEXICO STATE UNIV.

OREGON STATE UNIV.

\author{
STANFORD UNIVERSITY \\ UNIV. OF BRITISH COLUMBIA \\ UNIV. OF CALIFORNIA, BERKELEY \\ UNIV. OF CALIFORNIA, DAVIS \\ UNIV. OF CALIFORNIA, LOS ANGELES \\ UNIV. OF CALIFORNIA, RIVERSIDE \\ UNIV. OF CALIFORNIA, SAN DIEGO \\ UNIV. OF CALIF., SANTA BARBARA
}

\author{
Vyjayanthi Chari \\ Department of Mathematics \\ University of California \\ Riverside, CA 92521-0135 \\ chari@math.ucr.edu \\ Kefeng Liu \\ Department of Mathematics \\ University of California \\ Los Angeles, CA 90095-1555 \\ liu@math.ucla.edu \\ Jie Qing \\ Department of Mathematics \\ University of California \\ Santa Cruz, CA 95064 \\ qing@cats.ucsc.edu
}

These supporting institutions contribute to the cost of publication of this Journal, but they are not owners or publishers and have no responsibility for its contents or policies.

See inside back cover or msp.org/pjm for submission instructions.

The subscription price for 2013 is US \$400/year for the electronic version, and \$485/year for print and electronic.

Subscriptions, requests for back issues and changes of subscribers address should be sent to Pacific Journal of Mathematics, P.O. Box 4163, Berkeley, CA 94704-0163, U.S.A. The Pacific Journal of Mathematics is indexed by Mathematical Reviews, Zentralblatt MATH, PASCAL CNRS Index, Referativnyi Zhurnal, Current Mathematical Publications and the Science Citation Index.

The Pacific Journal of Mathematics (ISSN 0030-8730) at the University of California, c/o Department of Mathematics, 798 Evans Hall \#3840, Berkeley, CA 94720-3840, is published monthly except July and August. Periodical rate postage paid at Berkeley, CA 94704, and additional mailing offices. POSTMASTER: send address changes to Pacific Journal of Mathematics, P.O. Box 4163, Berkeley, CA 94704-0163.

PJM peer review and production are managed by EditFLOW ${ }^{\circledR}$ from Mathematical Sciences Publishers.

PUBLISHED BY

mathematical sciences publishers

nonprofit scientific publishing

http://msp.org/

(C) 2013 Mathematical Sciences Publishers 


\section{PACIFIC JOURNAL OF MATHEMATICS}

Volume $263 \quad$ No. $1 \quad$ May 2013

Biharmonic hypersurfaces in complete Riemannian manifolds

Luis J. Alías, S. Carolina García-Martínez and Marco

RIGOLI

Half-commutative orthogonal Hopf algebras

JULIEN BiCHON and MiCHEL DUBOIS-ViOLETTE

Superdistributions, analytic and algebraic super Harish-Chandra pairs

Claudio CARMELI and RITA FIORESI

Orbifolds with signature $\left(0 ; k, k^{n-1}, k^{n}, k^{n}\right)$

Angel Carocca, Rubén A. Hidalgo and Rubí E.

RODRÍGUEZ

Explicit isogeny theorems for Drinfeld modules

IMIN CHEN and YOONJIN LEE

Topological pressures for $\epsilon$-stable and stable sets

XiAnfeng Ma and ERCAi Chen

Lipschitz and bilipschitz maps on Carnot groups

WILLIAM MEYERSON

Geometric inequalities in Carnot groups

FRANCESCOPAOLO MONTEFALCONE

Fixed points of endomorphisms of virtually free groups

PEDRO V. SILVA

The sharp lower bound for the first positive eigenvalue of the

Folland-Stein operator on a closed pseudohermitian $(2 n+1)$-manifold

CHIN-TUNG Wu

Remark on "Maximal functions on the unit $n$-sphere" by Peter M. Knopf 253 (1987)

HONG-QUAN LI 